\title{
Inverse problems for linear hyperbolic equations using mixed formulations
}

\author{
Nicolae CîndeA* ARnaud $\mathrm{MÜNCH}^{\dagger}$
}

September 18, 2018

\begin{abstract}
We introduce in this document a direct method allowing to solve numerically inverse type problems for linear hyperbolic equations. We first consider the reconstruction of the full solution of the wave equation posed in $\Omega \times(0, T)$ - $\Omega$ a bounded subset of $\mathbb{R}^{N}$ - from a partial distributed observation. We employ a least-squares technique and minimize the $L^{2}$-norm of the distance from the observation to any solution. Taking the hyperbolic equation as the main constraint of the problem, the optimality conditions are reduced to a mixed formulation involving both the state to reconstruct and a Lagrange multiplier. Under usual geometric optic conditions, we show the well-posedness of this mixed formulation (in particular the inf-sup condition) and then introduce a numerical approximation based on space-time finite elements discretization. We prove the strong convergence of the approximation and then discussed several examples for $N=1$ and $N=2$. The problem of the reconstruction of both the state and the source term is also addressed.
\end{abstract}

KEYWORDS :Linear wave equation, Inverse problem, Finite elements methods; Mixed formulation

AMS NUMBER: 35L10, 65M12, 93B40.

\section{Introduction - Inverse problems for hyperbolic equations}

Let $\Omega$ be a bounded domain of $\mathbb{R}^{N}(N \geq 1)$ whose boundary $\partial \Omega$ is Lipschitz and let $T>0$. We note $Q_{T}:=\Omega \times(0, T)$ and $\Sigma_{T}:=\partial \Omega \times(0, T)$. We are concerned in this work with inverse type problems for linear hyperbolic equation of the following type

$$
\begin{cases}y_{t t}-\nabla \cdot(c(x) \nabla y)+d(x, t) y=f, & (x, t) \in Q_{T} \\ y=0, & (x, t) \in \Gamma_{T} \\ \left(y(\cdot, 0), y_{t}(\cdot, 0)\right)=\left(y_{0}, y_{1}\right), & x \in \Omega .\end{cases}
$$

We assume that $c \in C^{1}(\bar{\Omega}, \mathbb{R})$ with $c(x) \geq c_{0}>0$ in $\bar{\Omega}, d \in L^{\infty}\left(Q_{T}\right),\left(y_{0}, y_{1}\right) \in \boldsymbol{H}:=L^{2}(\Omega) \times$ $H^{-1}(\Omega)$ and $f \in X:=L^{2}\left(0, T ; H^{-1}(\Omega)\right)$.

For any $\left(y_{0}, y_{1}\right) \in \boldsymbol{H}$ and any $f \in X$, there exists exactly one solution $y$ to (1), with $y \in$ $C^{0}\left([0, T] ; L^{2}(\Omega)\right) \cap C^{1}\left([0, T] ; H^{-1}(\Omega)\right)($ see $[20])$.

In the sequel, for simplicity, we shall use the following notation:

$$
L y:=y_{t t}-\nabla \cdot(c(x) \nabla y)+d(x, t) y .
$$

* Laboratoire de Mathématiques, Université Blaise Pascal (Clermont-Ferrand 2), UMR CNRS 6620, Campus de Cézeaux, 63177, Aubière, France. E-mails: nicolae.cindea@math.univ-bpclermont.fr.

†Laboratoire de Mathématiques, Université Blaise Pascal (Clermont-Ferrand 2), UMR CNRS 6620, Campus de Cézeaux, 63177, Aubière, France. E-mail: arnaud.munch@math.univ-bpclermont.fr. 
and $X^{\prime}:=L^{2}\left(0, T ; H_{0}^{1}(\Omega)\right)$.

Let now $\omega$ be any non empty open subset of $\Omega$ and let $q_{T}:=\omega \times(0, T) \subset Q_{T}$. A typical inverse problem for 11 is the following one : from an observation or measurement $y_{o b s}$ in $L^{2}\left(q_{T}\right)$ on the sub-domain $q_{T}$, we want to recover a solution $y$ of the boundary value problem (1) which coincides with the observation on $q_{T}$.

Introducing the operator $P: L^{2}\left(Q_{T}\right) \rightarrow X \times L^{2}\left(q_{T}\right)$ defined by $P$ y $:=\left(L y, y_{\mid q_{T}}\right)$, the problem is reformulated as :

$$
\text { find } y \in L^{2}\left(Q_{T}\right) \text { solution of } P y=\left(f, y_{o b s}\right) \text {. }
$$

From the unique continuation property for (1), if the set $q_{T}$ satisfies some geometric conditions and if $y_{o b s}$ is a restriction to $q_{T}$ of a solution of (1), then the problem is well-posed in the sense that the state $y$ corresponding to the pair $\left(y_{o b s}, f\right)$ is unique.

In view of the unavoidable uncertainties on the data $y_{o b s}$ (coming from measurements, numerical approximations, etc), the problem needs to be relaxed. In this respect, the most natural (and widely used in practice) approach consists to introduce the following extremal problem (of least-squares type)

$$
\left\{\begin{array}{l}
\text { minimize over } \boldsymbol{H} \quad J\left(y_{0}, y_{1}\right):=\frac{1}{2}\left\|y-y_{o b s}\right\|_{L^{2}\left(q_{T}\right)}^{2} \\
\text { where } y \text { solves (1), }
\end{array}\right.
$$

since $y$ is uniquely and fully determined from $f$ and the data $\left(y_{0}, y_{1}\right)$. Here the constraint $y-y_{o b s}=$ 0 in $L^{2}\left(q_{T}\right)$ is relaxed; however, if $y_{o b s}$ is a restriction to $q_{T}$ of a solution of (1), then problems (LS) and $I P$ obviously coincide. A minimizing sequence for $J$ in $\boldsymbol{H}$ is easily defined in term of the solution of an auxiliary adjoint problem. Apart from a possible low decrease of the sequence near extrema, the main drawback, when one wants to prove the convergence of a discrete approximation is that, it is in general not possible to minimize over a discrete subspace of $\{y ; L y-f=0\}$ subject to the equality (in $X$ ) $L y-f=0$. Therefore, the minimization procedure first requires the discretization of the functional $J$ and of the system (1); this raised the issue of uniform coercivity property (typically here some uniform discrete observability inequality for the adjoint solution) of the discrete functional with respect to the approximation parameter. As far as we know, this delicate issue has received answers only for specific and somehow academic situations (uniform Cartesian approximation of $\Omega$, constant coefficients in (1)). We refer to [12, 17, 19, 22] and the references therein.

More recently, a different method to solve inverse type problems like $I P$ has emerged and use so called Luenberger type observers: this consists in defining, from the observation on $q_{T}$, an auxiliary boundary value problem whose solution possesses the same asymptotic behavior in time than the solution of (1): the use of the reversibility of the hyperbolic equation then allows to reconstruct the initial data $\left(y_{0}, y_{1}\right)$. We refer to [8, 24] and the references therein. But, for the same reasons, on a numerically point of view, these method require to prove uniform discrete observability properties.

In a series of works, Klibanov and co-workers use different approaches to solve inverse problems (we refer to [18] and the references therein): they advocate in particular the quasi-reversibility method which reads as follows : for any $\varepsilon>0$, find $y_{\varepsilon} \in \mathcal{A}$ the solution of

$$
\left\langle P y_{\varepsilon}, P \bar{y}\right\rangle_{X \times L^{2}\left(q_{T}\right)}+\varepsilon\left\langle y_{\varepsilon}, \bar{y}\right\rangle_{\mathcal{A}}=\left\langle\left(f, y_{o b s}\right), P \bar{y}\right\rangle_{X^{\prime} \times L^{2}\left(q_{T}\right), X \times L^{2}\left(q_{T}\right)},
$$

for all $\bar{y} \in \mathcal{A}$, where $\mathcal{A}$ denotes a Hilbert space subset of $L^{2}\left(Q_{T}\right)$ so that $P y \in X \times L^{2}\left(q_{T}\right)$ for all $y \in \mathcal{A}$ and $\varepsilon>0$ a Tikhonov like parameter which ensures the well-posedness. We refer for instance to [13] where the lateral Cauchy problem for the wave equation with non constant diffusion is addressed within this method. Remark that $(Q R)$ can be viewed as a least-squares problem since the solution $y_{\varepsilon}$ minimizes over $\mathcal{A}$ the functional $y \rightarrow\left\|P y-\left(f, y_{o b s}\right)\right\|_{X \times L^{2}\left(q_{T}\right)}^{2}+\varepsilon\|y\|_{\mathcal{A}}^{2}$. Eventually, if $y_{o b s}$ is a restriction to $q_{T}$ of a solution of (1), the corresponding $y_{\varepsilon}$ converges in $L^{2}\left(Q_{T}\right)$ toward 
to the solution of $(I P]$ as $\varepsilon \rightarrow 0$. There, unlike in Problem $[L S]$, the unknown is the state variable $y$ itself (as it is natural for elliptic equations) so that any standard numerical methods based on a conformal approximation of the space $\mathcal{A}$ together with appropriate observability inequalities allow to obtain a convergent approximation of the solution. In particular, there is no need to prove discrete observability inequalities. We refer to the book [2]. We also mention [6, 5] where a similar technique has been used recently to solve the inverse obstacle problem associated to the Laplace equation, which consists in finding an interior obstacle from boundary Cauchy data.

In the spirit of the works [18, 6, 13, we explore the direct resolution of the optimality conditions associated to the extremal problem $(\overline{L S})$, without Tikhonov parameter while keeping $y$ as the unknown of the problem. This strategy, which avoids any iterative process, has been successfully applied in the closed context of the exact controllability of (1) in [12] and 17, 10. The idea is to take into account the state constraint $L y-f=0$ with a Lagrange multiplier. This allows to derive explicitly the optimality systems associated to $L S$ in term of an elliptic mixed formulation and therefore reformulate the original problem. Well-posedness of such new formulation is related to an observability inequality for the homogeneous solution of the hyperbolic equation.

The outline of this paper is as follow. In Section 2, we consider the least-squares problem $(\mathcal{P})$ and reconstruct the solution of the wave equation from a partial observation localized on a subset $q_{T}$ of $Q_{T}$. For that, in Section 2.1, we associate to $(\mathcal{P}$ the equivalent mixed formulation (7) which relies on the optimality conditions of the problem. Assuming that $q_{T}$ satisfies the classical geometric optic condition (Hypothesis 1, see $(\mathcal{H})$ ), we then show the well-posedness of this mixed formulation, in particular, we check the Babuska-Brezzi inf-sup condition (see Theorem 2.1). Interestingly, in Section 2.2, we also derive a equivalent dual extremal problem, which reduces the determination of the state $y$ to the minimization of an elliptic functional with respect to the Lagrange multiplier. In Section 3, we apply the same procedure to recover from a partial observation both the state and the source term. Section 4 is devoted to the numerical approximation, through a conformal space-time finite element discretization. The strong convergence of the approximation $\left(y_{h}, f_{h}\right)$ is shown as the discretization parameter $h$ tends to zero. In particular, we discuss the discrete inf-sup property of the mixed formulation. We present numerical experiments in Section 5 for $\Omega=(0,1)$ and $\Omega \subset \mathbb{R}^{2}$, in agreement with the theoretical part. We consider in particular time dependent observation zones. Section 6 concludes with some perspectives.

\section{Recovering the solution from a partial observation: a mixed re-formulation of the problem}

In this section, assuming that the initial $\left(y_{0}, y_{1}\right) \in \boldsymbol{H}$ are unknown, we address the inverse problem (IP). Without loss of generality, in view of the linearity of the system (1), we assume that the source term $f \equiv 0$.

We consider the non empty vectorial space $Z$ defined by

$$
Z:=\left\{y: y \in C\left([0, T], L^{2}(\Omega)\right) \cap C^{1}\left([0, T], H^{-1}(\Omega)\right), L y \in X\right\} .
$$

and then introduce the following hypothesis :

Hypothesis 1 There exists a constant $C_{o b s}=C\left(\omega, T,\|c\|_{C^{1}(\bar{\Omega})},\|d\|_{L^{\infty}(\Omega)}\right)$ such that the following estimate holds :

$$
\left\|y(\cdot, 0), y_{t}(\cdot, 0)\right\|_{\boldsymbol{H}}^{2} \leq C_{o b s}\left(\|y\|_{L^{2}\left(q_{T}\right)}^{2}+\|L y\|_{X}^{2}\right), \quad \forall y \in Z .
$$

Condition $(\mathcal{H})$ is a generalized observability inequality for the solution of the hyperbolic equation: for constant coefficients, this estimate is known to hold if the triplet $(\omega, T, \Omega)$ satisfies a 
geometric optic condition. We refer to [1]. In particular, $T$ should be large enough. Upon the

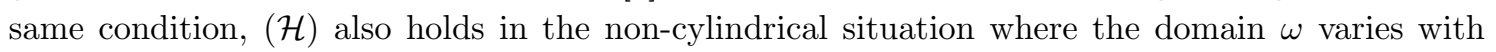
respect to the time variable: we refer to [7] for the one dimensional case. For non constant velocity $c$ and potential $d$, we refer to [10] and the references therein.

Then, within this hypothesis, for any $\eta>0$, we define on $Z$ the bilinear form

$$
\langle y, \bar{y}\rangle_{Z}:=\iint_{q_{T}} y \bar{y} d x d t+\eta \int_{0}^{T}\langle L y, L \bar{y}\rangle_{H^{-1}(\Omega)} d t \quad \forall y, \bar{y} \in Z .
$$

In view of $(\mathcal{H})$, this bilinear form defines a scalar product over $Z$. Moreover, endowed to this scalar product, we easily obtain that $Z$ is a Hilbert space (see [7, Corollary 2.4). We note the corresponding norm by $\|y\|_{Z}:=\sqrt{\langle y, y\rangle_{Z}}$.

Then, we consider the following extremal problem :

$$
\left\{\begin{array}{l}
\inf J(y):=\frac{1}{2}\left\|y-y_{o b s}\right\|_{L^{2}\left(q_{T}\right)}^{2}, \\
\text { subject to } \quad y \in W
\end{array}\right.
$$

where $W$ is the closed subspace of $Z$ defined by

$$
W:=\{y \in Z ; L y=0 \text { in } X\}
$$

and endowed with the norm of $Z$.

The extremal problem $\sqrt{\mathcal{P}}$ is well posed : the functional $J$ is continuous over $W$, is strictly convex and is such that $J(y) \rightarrow+\infty$ as $\|y\|_{W} \rightarrow \infty$. Note also that the solution of $(\mathcal{P})$ in $W$ does not depend on $\eta$.

Remind that from the definition of $Z, L y$ belongs to $X$. Similarly, the uniqueness of the solution is lost if the hypothesis $(\mathcal{H})$ is not fulfilled, for instance if $T$ is not large enough. Eventually, from $(\mathcal{H})$, the solution $y$ in $Z$ of $(\mathcal{P})$ satisfies $\left(y(\cdot, 0), y_{t}(\cdot, 0)\right) \in \boldsymbol{H}$, so that problem $\mathcal{P}$ is equivalent to the minimization of $J$ with respect to $\left(y_{0}, y_{1}\right) \in \boldsymbol{H}$ as in problem $(I P)$, Section 1 .

We also recall that for any $z \in Z$ there exists a positive constant $C_{\Omega, T}$ such that

$$
\|z\|_{L^{2}\left(Q_{T}\right)}^{2} \leq C_{\Omega, T}\left(\left\|z(\cdot, 0), z_{t}(\cdot, 0)\right\|_{\boldsymbol{H}}^{2}+\|L z\|_{X}^{2}\right) .
$$

This equality and $(\mathcal{H})$ imply that

$$
\|z\|_{L^{2}\left(Q_{T}\right)}^{2} \leq C_{\Omega, T}\left(C_{o b s}\|z\|_{L^{2}\left(q_{T}\right)}^{2}+\left(1+C_{o b s}\right)\|L z\|_{X}^{2}\right), \quad \forall z \in Z .
$$

\subsection{Direct approach}

In order to solve $(\mathcal{P})$, we have to deal with the constraint equality which appears in the space $W$. Proceeding as in [12, we introduce a Lagrangian multiplier $\lambda \in X^{\prime}$ and the following mixed formulation: find $(y, \lambda) \in Z \times X^{\prime}$ solution of

$$
\left\{\begin{aligned}
a(y, \bar{y})+b(\bar{y}, \lambda) & =l(\bar{y}), & & \forall \bar{y} \in Z \\
b(y, \bar{\lambda}) & =0, & & \forall \bar{\lambda} \in X^{\prime},
\end{aligned}\right.
$$

where

$$
\begin{aligned}
& a: Z \times Z \rightarrow \mathbb{R}, \quad a(y, \bar{y}):=\iint_{q_{T}} y \bar{y} d x d t \\
& b: Z \times X^{\prime} \rightarrow \mathbb{R}, \quad b(y, \lambda):=\int_{0}^{T}\langle\lambda, L y\rangle_{H_{0}^{1}(\Omega), H^{-1}(\Omega)} d t, \\
& l: Z \rightarrow \mathbb{R}, \quad l(y):=\iint_{q_{T}} y_{o b s} y d x d t .
\end{aligned}
$$


System (7) is nothing else than the optimality system corresponding to the extremal problem $(\mathcal{P})$. Precisely, the following result holds :

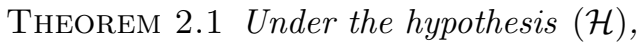

(i) The mixed formulation (7) is well-posed.

(ii) The unique solution $(y, \lambda) \in Z \times X^{\prime}$ to (7) is the unique saddle-point of the Lagrangian $\mathcal{L}: Z \times X^{\prime} \rightarrow \mathbb{R}$ defined by

$$
\mathcal{L}(y, \lambda):=\frac{1}{2} a(y, y)+b(y, \lambda)-l(y)
$$

(iii) We have the estimate

$$
\|y\|_{Z}=\|y\|_{L^{2}\left(q_{T}\right)} \leq\left\|y_{o b s}\right\|_{L^{2}\left(q_{T}\right)}, \quad\|\lambda\|_{X^{\prime}} \leq 2 \sqrt{C_{\Omega, T}+\eta}\left\|y_{o b s}\right\|_{L^{2}\left(q_{T}\right)} .
$$

Proof- We use classical results for saddle point problems (see [4, chapter 4).

We easily obtain the continuity of the bilinear form $a$ over $Z \times Z$, the continuity of bilinear $b$ over $Z \times X^{\prime}$ and the continuity of the linear form $l$ over $Z$. In particular, we get

$$
\|l\|_{Z^{\prime}}=\left\|y_{o b s}\right\|_{L^{2}\left(q_{T}\right)}, \quad\|a\|_{(Z \times Z)^{\prime}}=1, \quad\|b\|_{\left(Z \times X^{\prime}\right)^{\prime}}=\eta^{-1 / 2} .
$$

Moreover, the kernel $\mathcal{N}(b)=\left\{y \in Z ; b(y, \lambda)=0 \quad \forall \lambda \in X^{\prime}\right\}$ coincides with $W$ : we easily get

$$
a(y, y)=\|y\|_{Z}^{2}, \quad \forall y \in \mathcal{N}(b)=W .
$$

Therefore, in view of [4, Theorem 4.2.2], it remains to check the inf-sup constant property : $\exists \delta>0$ such that

$$
\inf _{\lambda \in X^{\prime}} \sup _{y \in Z} \frac{b(y, \lambda)}{\|y\|_{Z}\|\lambda\|_{X^{\prime}}} \geq \delta .
$$

We proceed as follows. For any fixed $\lambda \in X^{\prime}$, we define $y$ as the unique solution of

$$
L y=-\Delta \lambda \text { in } Q_{T}, \quad\left(y(\cdot, 0), y_{t}(\cdot, 0)\right)=(0,0) \text { on } \Omega, \quad y=0 \text { on } \Sigma_{T} .
$$

We get $b(y, \lambda)=\|\lambda\|_{X^{\prime}}^{2}$ and

$$
\|y\|_{Z}^{2}=\|y\|_{L^{2}\left(q_{T}\right)}^{2}+\eta\|\lambda\|_{X^{\prime}}^{2} .
$$

Using [5, the estimate $\|y\|_{L^{2}\left(q_{T}\right)} \leq \sqrt{C_{\Omega, T}}\|\lambda\|_{X^{\prime}}$ implies that $y \in Z$ and that

$$
\sup _{y \in Z} \frac{b(y, \lambda)}{\|y\|_{Z}\|\lambda\|_{X^{\prime}}} \geq \frac{1}{\sqrt{C_{\Omega, T}+\eta}}>0
$$

leading to the result with $\delta=\left(C_{\Omega, T}+\eta\right)^{-1 / 2}$.

The third point is the consequence of classical estimates (see [4, Theorem 4.2.3.) :

$$
\|y\|_{Z} \leq \frac{1}{\alpha_{0}}\|l\|_{Z^{\prime}}, \quad\|\lambda\|_{X^{\prime}} \leq \frac{1}{\delta}\left(1+\frac{\|a\|}{\alpha_{0}}\right)\|l\|_{Z^{\prime}}
$$

where

$$
\alpha_{0}:=\inf _{y \in \mathcal{N}(b)} \frac{a(y, y)}{\|y\|_{Z}^{2}} .
$$

Estimates 12 and the equality $\alpha_{0}=1$ lead to the results. Eventually, from 12 , we obtain that

$$
\|\lambda\|_{X^{\prime}} \leq \frac{2}{\delta}\left\|y_{o b s}\right\|_{L^{2}\left(q_{T}\right)}
$$


and that $\delta \geq\left(C_{\Omega, T}+\eta\right)^{-1 / 2}$ to get 11 .

In practice, it is very convenient to "augment" the Lagrangian (see [16]) and consider instead the Lagrangian $\mathcal{L}_{r}$ defined for any $r>0$ by

$$
\begin{aligned}
& \mathcal{L}_{r}(y, \lambda):=\frac{1}{2} a_{r}(y, y)+b(y, \lambda)-l(y), \\
& a_{r}(y, y):=a(y, y)+r\|L y\|_{X}^{2} .
\end{aligned}
$$

Since $a_{r}(y, y)=a(y, y)$ on $W$, the Lagrangian $\mathcal{L}$ and $\mathcal{L}_{r}$ share the same saddle-point. The positive number $r$ is an augmentation parameter.

Remark 1 Assuming additional hypotheses on the regularity of the solution $\lambda$, precisely $L \lambda \in$ $L^{2}\left(Q_{T}\right)$ and $\left(\lambda, \lambda_{t}\right)_{\mid t=0, T} \in H_{0}^{1}(\Omega) \times L^{2}(\Omega)$, we easily prove, writing the optimality condition for $\mathcal{L}$, that the multiplier $\lambda$ satisfies the following relations :

$$
\left\{\begin{array}{l}
L \lambda=-\left(y-y_{\text {obs }}\right) 1_{\omega} \text { in } \quad Q_{T}, \quad \lambda=0 \quad \text { in } \Sigma_{T}, \\
\lambda=\lambda_{t}=0 \text { on } \Omega \times\{0, T\} .
\end{array}\right.
$$

Therefore, $\lambda$ (defined in the weak sense) is an exact controlled solution of the wave equation through the control $-\left(y-y_{o b s}\right) 1_{\omega} \in L^{2}\left(q_{T}\right)$.

- If $y_{o b s}$ is the restriction to $q_{T}$ of a solution of (1), then the unique multiplier $\lambda$ must vanish almost everywhere. In that case, we have $\sup _{\lambda \in \Lambda} \inf _{y \in Y} \mathcal{L}_{r}(y, \lambda)=\inf _{y \in Y} \mathcal{L}_{r}(y, 0)=$ $\inf _{y \in Y} J_{r}(y)$ with

$$
J_{r}(y):=\frac{1}{2}\left\|y-y_{o b s}\right\|_{L^{2}\left(Q_{T}\right)}^{2}+\frac{r}{2}\|L y\|_{X}^{2} .
$$

The corresponding variational formulation is then : find $y \in Z$ such that

$$
a_{r}(y, \bar{y})=\iint_{q_{T}} y \bar{y} d x d t+r \int_{0}^{T}\langle L y, L \bar{y}\rangle_{H^{-1}(\Omega)} d t=l(\bar{y}), \quad \forall \bar{y} \in Z .
$$

- In the general case, the mixed formulation can be rewritten as follows: find $(z, \lambda) \in Z \times X^{\prime}$ solution of

$$
\left\{\begin{aligned}
\left\langle P_{r} y, P_{r} \bar{y}\right\rangle_{X \times L^{2}\left(q_{T}\right)}+\langle L \bar{y}, \lambda\rangle_{X, X^{\prime}} & =\left\langle\left(0, y_{o b s}\right), P_{r} \bar{y}\right\rangle_{X \times L^{2}\left(q_{T}\right)}, \quad \forall \bar{y} \in Z, \\
\langle L \bar{y}, \lambda\rangle_{X, X^{\prime}} & =0, \quad \forall \lambda \in X^{\prime}
\end{aligned}\right.
$$

with $P_{r} y:=\left(\sqrt{r} L y, y_{\mid q_{T}}\right)$. This approach may be seen as generalization of the $Q R$ problem $($ see $(Q R))$, where the variable $\lambda$ is adjusted automatically (while the choice of the parameter $\varepsilon$ in $(Q R)$ is in general a delicate issue).

System (16) can be used to define a equivalent saddle-point formulation, very suitable at the numerical level. Precisely, we introduce - in view of $(16)$ - the space $\Lambda$ by

$$
\begin{aligned}
\Lambda:=\{\lambda: \lambda \in C([0, T] ; & \left.H_{0}^{1}(\Omega)\right) \cap C^{1}\left([0, T] ; L^{2}(\Omega)\right), \\
& \left.L \lambda \in L^{2}\left(Q_{T}\right), \lambda(\cdot, 0)=\lambda_{t}(\cdot, 0)=0\right\} .
\end{aligned}
$$

Endowed with the scalar product $\langle\lambda, \bar{\lambda}\rangle_{\Lambda}:=\iint_{Q_{T}}(\lambda \bar{\lambda}+L \lambda L \bar{\lambda}) d x d t$, we check that $\Lambda$ is a Hilbert space. Then, for any parameter $\alpha \in(0,1)$, we consider the following mixed formulation : find $(y, \lambda) \in Z \times \Lambda$ such that 


$$
\left\{\begin{aligned}
a_{r, \alpha}(y, \bar{y})+b_{\alpha}(\bar{y}, \lambda) & =l_{1, \alpha}(\bar{y}), & & \forall \bar{y} \in Z \\
b_{\alpha}(y, \bar{\lambda})-c_{\alpha}(\lambda, \bar{\lambda}) & =l_{2, \alpha}(\bar{\lambda}), & & \forall \bar{\lambda} \in \Lambda,
\end{aligned}\right.
$$

where

$$
\begin{aligned}
& a_{r, \alpha}: Z \times Z \rightarrow \mathbb{R}, \quad a_{r, \alpha}(y, \bar{y}):=(1-\alpha) \iint_{q_{T}} y \bar{y} d x d t+r \int_{0}^{T}(L y, L \bar{y})_{H^{-1}(\Omega)} d t, \\
& b_{\alpha}: Z \times \Lambda \rightarrow \mathbb{R}, \quad b_{\alpha}(y, \lambda):=\int_{0}^{T}\langle\lambda, L y\rangle_{H_{0}^{1}(\Omega), H^{-1}(\Omega)} d t-\alpha \iint_{q_{T}} y L \lambda d x d t \\
& c_{\alpha}: \Lambda \times \Lambda \rightarrow \mathbb{R}, \quad c_{\alpha}(\lambda, \bar{\lambda}):=\alpha \iint_{Q_{T}} L \lambda L \bar{\lambda}, d x d t \\
& l_{1, \alpha}: Z \rightarrow \mathbb{R}, \quad l_{1, \alpha}(y):=(1-\alpha) \iint_{q_{T}} y_{o b s} y d x d t \\
& l_{2, \alpha}: \Lambda \rightarrow \mathbb{R}, \quad l_{2, \alpha}(\lambda):=-\alpha \iint_{q_{T}} y_{o b s} L \lambda d x d t .
\end{aligned}
$$

From the symmetry of $a_{r, \alpha}$ and $c_{\alpha}$, we easily check that this formulation corresponds to the saddle point problem :

$$
\left\{\begin{array}{l}
\sup _{\lambda \in \Lambda} \inf _{y \in Z} \mathcal{L}_{r, \alpha}(y, \lambda), \\
\mathcal{L}_{r, \alpha}(y, \lambda):=\mathcal{L}_{r}(y, \lambda)-\frac{\alpha}{2}\left\|L \lambda+\left(y-y_{o b s}\right) 1_{\omega}\right\|_{L^{2}\left(Q_{T}\right)}^{2}
\end{array}\right.
$$

Proposition 2.1 Under the hypothesis (H), for any $\alpha \in(0,1)$, the formulation (19) is well-posed. Moreover, the unique pair $(y, \lambda)$ in $Z \times \Lambda$ satisfies

$$
\theta_{1}\|y\|_{Z}^{2}+\theta_{2}\|\lambda\|_{\Lambda}^{2} \leq\left(\frac{(1-\alpha)^{2}}{\theta_{1}}+\frac{\alpha^{2}}{\theta_{2}}\right)\left\|y_{o b s}\right\|_{L^{2}\left(q_{T}\right)}^{2} .
$$

with

$$
\theta_{1}:=\min \left(1-\alpha, \frac{r}{\eta}\right), \quad \theta_{2}:=\frac{1}{2} \min \left(\alpha, \frac{1}{C_{\Omega, T}}\right) .
$$

Proof- We easily get the continuity of the bilinear forms $a_{r, \alpha}, b_{\alpha}$ and $c_{\alpha}$ :

$$
\begin{aligned}
& \left|a_{r, \alpha}(y, \bar{y})\right| \leq \max \left(1-\alpha, \frac{r}{\eta}\right)\|y\|_{Z}\|\bar{y}\|_{Z}, \quad \forall y, \bar{y} \in Z, \\
& \left|b_{\alpha}(y, \lambda)\right| \leq \max \left(\alpha, \frac{1}{\sqrt{\eta}}\right)\|y\|_{Z}\|\lambda\|_{\Lambda}, \quad \forall y \in Z, \forall \lambda \in \Lambda, \\
& \mid c_{\alpha}(\lambda, \bar{\lambda}) \leq \alpha\|\lambda\|_{\Lambda}\|\bar{\lambda}\|_{\Lambda}, \quad \forall \lambda, \bar{\lambda} \in \Lambda
\end{aligned}
$$

and of the linear form $l_{1}$ and $l_{2}:\left\|l_{1}\right\|_{Z^{\prime}}=(1-\alpha)\left\|y_{o b s}\right\|_{L^{2}\left(q_{T}\right)}$ and $\left\|l_{2}\right\|_{\Lambda^{\prime}}=\alpha\left\|y_{o b s}\right\|_{L^{2}\left(q_{T}\right)}$.

Moreover, since $\alpha \in(0,1)$, we also obtain the coercivity of $a_{r, \alpha}$ and of $c_{\alpha}$ : precisely,

$$
\begin{aligned}
& a_{r, \alpha}(y, y) \geq \min \left(1-\alpha, \frac{r}{\eta}\right)\|y\|_{Z}^{2}, \quad \forall y \in Z, \\
& c_{\alpha}(\lambda, \lambda) \geq \min \left(\alpha m, \frac{1-m}{C_{\Omega, T}}\right)\|\lambda\|_{\Lambda}^{2} \quad \forall \lambda \in \Lambda, \quad \forall m \in(0,1) .
\end{aligned}
$$

The result [4, Prop 4.3.1] implies the well-posedness and the estimate 20 taking $m=1 / 2$.

The $\alpha$-term in $\mathcal{L}_{r, \alpha}$ is a stabilization term: it ensures a coercivity property of $\mathcal{L}_{r, \alpha}$ with respect to the variable $\lambda$ and automatically the well-posedness. In particular, there is no need to prove any inf-sup property for the application $b_{\alpha}$. 
Proposition 2.2 If the solution $(y, \lambda) \in Z \times X^{\prime}$ of (7) enjoys the property $\lambda \in \Lambda$, then the solutions of (7) and (19) coincide.

Proof- The hypothesis of regularity and the relation 16 imply that the solution $(y, \lambda) \in Z \times X^{\prime}$ of $(7)$ is also a solution of $(19)$. The result then follows from the uniqueness of the two formulations.

\subsection{Dual formulation of the extremal problem (7)}

As discussed at length in [12, we may also associate to the extremal problem $(\mathcal{P}$ an equivalent problem involving only the variable $\lambda$. Again, this is particularly interesting at the numerical level. This requires a strictly positive augmentation parameter $r$.

For any $r>0$, let us define the linear operator $\mathcal{P}_{r}$ from $X^{\prime}$ into $X^{\prime}$ by

$$
\mathcal{P}_{r} \lambda:=-\Delta^{-1}(L y), \quad \forall \lambda \in X^{\prime}
$$

where $y \in Z$ is the unique solution to

$$
a_{r}(y, \bar{y})=b(\bar{y}, \lambda), \quad \forall \bar{y} \in Z .
$$

The assumption $r>0$ is necessary here in order to guarantee the well-posedness of 21]. Precisely, for any $r>0$, the form $a_{r}$ defines a norm equivalent to the norm on $Z$.

The following important lemma holds:

Lemma 2.1 For any $r>0$, the operator $\mathcal{P}_{r}$ is a strongly elliptic, symmetric isomorphism from $X^{\prime}$ into $X^{\prime}$.

Proof- From the definition of $a_{r}$, we easily get that $\left\|\mathcal{P}_{r} \lambda\right\|_{X^{\prime}} \leq r^{-1}\|\lambda\|_{X^{\prime}}$ and the continuity of $\mathcal{P}_{r}$. Next, consider any $\lambda^{\prime} \in X^{\prime}$ and denote by $y^{\prime}$ the corresponding unique solution of (21) so that $\mathcal{P}_{r} \lambda^{\prime}:=-\Delta^{-1}\left(L y^{\prime}\right)$. Relation 21] with $\bar{y}=y^{\prime}$ then implies that

$$
\int_{0}^{T}\left\langle\mathcal{P}_{r} \lambda^{\prime}, \lambda\right\rangle_{H_{0}^{1}(\Omega)} d t=a_{r}\left(y, y^{\prime}\right)
$$

and therefore the symmetry and positivity of $\mathcal{P}_{r}$. The last relation with $\lambda^{\prime}=\lambda$ and the observability estimate $(\mathcal{H})$ imply that $\mathcal{P}_{r}$ is also positive definite.

Finally, let us check the strong ellipticity of $\mathcal{P}_{r}$, equivalently that the bilinear functional $\left(\lambda, \lambda^{\prime}\right) \rightarrow \int_{0}^{T}\left\langle\mathcal{P}_{r} \lambda, \lambda^{\prime}\right\rangle_{H_{0}^{1}(\Omega), H_{0}^{1}(\Omega)} d t$ is $X^{\prime}$-elliptic. Thus we want to show that

$$
\int_{0}^{T}\left\langle\mathcal{P}_{r} \lambda, \lambda\right\rangle_{H_{0}^{1}(\Omega)} d t \geq C\|\lambda\|_{X^{\prime}}^{2}, \quad \forall \lambda \in X^{\prime}
$$

for some positive constant $C$. Suppose that 23) does not hold; there exists then a sequence $\left\{\lambda_{n}\right\}_{n \geq 0}$ of $X^{\prime}$ such that

$$
\left\|\lambda_{n}\right\|_{X^{\prime}}=1, \quad \forall n \geq 0, \quad \lim _{n \rightarrow \infty} \int_{0}^{T}\left\langle\mathcal{P}_{r} \lambda_{n}, \lambda_{n}\right\rangle_{H_{0}^{1}(\Omega)} d t=0 .
$$

Let us denote by $y_{n}$ the solution of 21) corresponding to $\lambda_{n}$. From 22, we then obtain that

$$
\lim _{n \rightarrow \infty} r\left\|L y_{n}\right\|_{X}^{2}+\left\|y_{n}\right\|_{L^{2}\left(q_{T}\right)}^{2}=0 .
$$

From 21) with $y=y_{n}$ and $\lambda=\lambda_{n}$, we have

$$
\int_{0}^{T}\left\langle r\left(-\Delta^{-1}\right) L y_{n}-\lambda_{n},\left(-\Delta^{-1}\right) L \bar{y}\right\rangle_{H_{0}^{1}(\Omega)} d t+\iint_{q_{T}} y_{n} \bar{y} d x d t=0, \quad \forall \bar{y} \in Z .
$$


We define the sequence $\left\{\bar{y}_{n}\right\}_{n \geq 0}$ as follows :

$$
\left\{\begin{array}{lll}
L \bar{y}_{n}=r L y_{n}+\Delta^{-1} \lambda_{n}, & \text { in } \quad Q_{T}, \\
\bar{y}_{n}=0, & \text { in } \quad \Sigma_{T}, \\
\bar{y}_{n}(\cdot, 0)=\bar{y}_{n, t}(\cdot, 0)=0, & \text { in } \Omega,
\end{array}\right.
$$

so that, for all $n, \bar{y}_{n}$ is the solution of the wave equation with zero initial data and source term $r L y_{n}+\Delta \lambda_{n}$ in $X$. Using again (5), we get $\left\|\bar{y}_{n}\right\|_{L^{2}\left(q_{T}\right)} \leq \sqrt{C_{\Omega, T}}\left\|r L y_{n}+\Delta \lambda_{n}\right\|_{X}$, so that $\bar{y}_{n} \in Z$. Then, using (25) with $\bar{y}=\bar{y}_{n}$ we get

$$
\left\|r\left(-\Delta^{-1}\right) L y_{n}-\lambda_{n}\right\|_{X^{\prime}} \leq \sqrt{C_{\Omega, T}}\left\|y_{n}\right\|_{L^{2}\left(q_{T}\right)} .
$$

Then, from (24), we conclude that $\lim _{n \rightarrow+\infty}\left\|\lambda_{n}\right\|_{X^{\prime}}=0$ leading to a contradiction and to the strong ellipticity of the operator $\mathcal{P}_{r}$.

The introduction of the operator $\mathcal{P}_{r}$ is motivated by the following proposition :

Proposition 2.3 For any $r>0$, let $y_{0} \in Z$ be the unique solution of

$$
a_{r}\left(y_{0}, \bar{y}\right)=l(\bar{y}), \quad \forall \bar{y} \in Z
$$

and let $J_{r}^{\star \star}: X^{\prime} \rightarrow X^{\prime}$ be the functional defined by

$$
J_{r}^{\star \star}(\lambda)=\frac{1}{2} \int_{0}^{T}\left\langle\mathcal{P}_{r} \lambda, \lambda\right\rangle_{H_{0}^{1}(\Omega)} d t-b\left(y_{0}, \lambda\right) .
$$

The following equality holds :

$$
\sup _{\lambda \in X^{\prime}} \inf _{y \in Z} \mathcal{L}_{r}(y, \lambda)=-\inf _{\lambda \in X^{\prime}} J_{r}^{\star \star}(\lambda) \quad+\mathcal{L}_{r}\left(y_{0}, 0\right) .
$$

The proof is classical and we refer for instance to [12 in a similar context. This proposition reduces the search of $y$, solution of problem $(\mathcal{P})$, to the minimization of $J_{r}^{\star \star}$. The well-posedness is a consequence of the ellipticity of the operator $\mathcal{P}_{r}$.

Remark 2 The results of this section apply if the distributed observation on $q_{T}$ is replaced by a Neumann boundary observation on a sufficiently large subset $\Sigma_{T}$ of $\partial \Omega \times(0, T)$ (i.e. assuming $\frac{\partial y}{\partial \nu}=$ $y_{o b s} \in L^{2}\left(\Sigma_{T}\right)$ is known on $\left.\Sigma_{T}\right)$. This is due to the following generalized observability inequality: there exists a positive constant $C_{o b s}=C\left(\omega, T,\|c\|_{C^{1}(\bar{\Omega})},\|d\|_{L^{\infty}(\Omega)}\right)$ such that the following estimate holds :

$$
\left\|y(\cdot, 0), y_{t}(\cdot, 0)\right\|_{H_{0}^{1}(\Omega) \times L^{2}(\Omega)}^{2} \leq C_{o b s}\left(\left\|\frac{\partial y}{\partial \nu}\right\|_{L^{2}\left(\Sigma_{T}\right)}^{2}+\|L y\|_{L^{2}\left(Q_{T}\right)}^{2}\right), \quad \forall y \in Z
$$

which holds if the triplet $\left(Q_{T}, \Sigma_{T}, T\right)$ satisfies the geometric condition as before (we refer to [10] and the references therein). Actually, it suffices to re-define the form a in (8) by $a(y, \bar{y}):=$ $\iint_{\Sigma_{T}} \frac{\partial y}{\partial \nu} \frac{\partial \bar{y}}{\partial \nu} d \sigma d x$ and the form $l$ by $l(y):=\iint_{\Sigma_{T}} \frac{\partial y}{\partial \nu} y_{o b s} d \sigma d x$ for all $y, \bar{y} \in Z$.

Remark 3 We emphasize that the mixed formulation (7) has a structure very closed to the one we get when we address - using the same approach - the null controllability of (1): more precisely, the control of minimal $L^{2}\left(q_{T}\right)$-norm which drives to rest the initial data $\left(y_{0}, y_{1}\right) \in H_{0}^{1}(\Omega) \times L^{2}(\Omega)$ is given by $v=\varphi 1_{q_{T}}$ where $(\varphi, \lambda) \in \Phi \times L^{2}\left(0, T ; H_{0}^{1}(\Omega)\right)$ solves the mixed formulation

$$
\left\{\begin{aligned}
a(\varphi, \bar{\varphi})+b(\bar{\varphi}, \lambda) & =l(\bar{\varphi}), & & \forall \bar{\varphi} \in \Phi \\
b(\varphi, \bar{\lambda}) & =0, & & \forall \bar{\lambda} \in L^{2}\left(0, T ; H_{0}^{1}(\Omega)\right)
\end{aligned}\right.
$$


where

$$
\begin{aligned}
& a: \Phi \times \Phi \rightarrow \mathbb{R}, \quad a(\varphi, \bar{\varphi})=\iint_{q_{T}} \varphi(x, t) \bar{\varphi}(x, t) d x d t \\
& b: \Phi \times L^{2}\left(0, T ; H_{0}^{1}(\Omega)\right) \rightarrow \mathbb{R}, \quad b(\varphi, \lambda)=\int_{0}^{T}\langle L \varphi, \lambda\rangle_{H^{-1}, H_{0}^{1}} d t \\
& l: \Phi \rightarrow \mathbb{R}, \quad l(\varphi)=-\left\langle\varphi_{t}(\cdot, 0), y_{0}\right\rangle_{H^{-1}(\Omega), H_{0}^{1}(\Omega)}+\int_{0}^{1} \varphi(\cdot, 0) y_{1} d x .
\end{aligned}
$$

with $\Phi=\left\{\varphi \in L^{2}\left(Q_{T}\right), \varphi=0\right.$ on $\Sigma_{T}$ such that $\left.L \varphi \in L^{2}\left(0, T ; H^{-1}(\Omega)\right)\right\}$. We refer to [12].

Remark 4 Reversing the order of priority between the constraint $y-y_{o b s}=0$ in $L^{2}\left(q_{T}\right)$ and $L y-f=0$ in $X$, a possibility could be to minimize the functional $y \rightarrow\|L y-f\|_{X}$ over $y \in Z$ subject to the constraint $y-y_{o b s}=0$ in $L^{2}\left(q_{T}\right)$ via the introduction of a Lagrange multiplier in $L^{2}\left(q_{T}\right)$. The proof of the following inf-sup property : there exists $\delta>0$ such that

$$
\inf _{\lambda \in L^{2}\left(q_{T}\right)} \sup _{y \in Z} \frac{\iint_{q_{T}} \lambda y d x d t}{\|\lambda\|_{L^{2}\left(q_{T}\right)}\|y\|_{Y}} \geq \delta
$$

associated to the corresponding mixed-formulation is however unclear. If a $\varepsilon$-term is added as in (QR), this difficulty disappears (we refer again to the book [18]).

\section{Recovering the source and the solution from a partial ob- servation: a mixed re-formulation of the problem}

Given a partial observation $y_{o b s}$ of the solution on the subset $q_{T} \subset Q_{T}$, we now consider the reconstruction of the full solution as well as the source term $f$ assumed in $X$. We assume that the initial data $\left(y_{0}, y_{1}\right) \in \boldsymbol{H}$ are unknown.

The situation is different with respect to the previous section, since without additional assumption on $f$, the couple $(y, f)$ is not unique. Consider the case of a source $f$ supported in a set which is near $\partial \Omega \times(0, T)$ and disjoint from $q_{T}$ : from the finite propagation of the solution, the source $f$ will not affect the solution $y$ in $q_{T}$. On the other hand, the determination of a couple $(y, f)$ which solves (1) such that $y$ coincides with $y_{o b s}$ is straightforward : it suffices to "extend" $y$ on $Q_{T} \backslash q_{T}$ appropriately to preserve the boundary conditions, then compute $L y$ and recover a source term. However, we emphasize that, on a practical viewpoint, the extension of $y_{o b s}$ out of $q_{T}$ is not obvious. Moreover, this strategy does not offer any control on the object $f$.

We briefly show that we can apply the method developed in Section 2 which allows a robust reconstruction and then consider the case of uniqueness via additional condition on $f$.

We assume again that $(\mathcal{H}$ holds. We note $Y:=Z \times X$ and define on $Y$ the bilinear form, for any $\varepsilon, \eta>0$

$$
\begin{aligned}
\langle(y, f),(\bar{y}, \bar{f})\rangle_{Y}:= & \iint_{q_{T}} y \bar{y} d x d t+\eta \int_{0}^{T}\langle L y-f, L \bar{y}-f\rangle_{H^{-1}(\Omega)} d t \\
& +\varepsilon \int_{0}^{T}\langle f, \bar{f}\rangle_{H^{-1}(\Omega)} d t, \quad \forall(y, f),(\bar{y}, \bar{f}) \in Y .
\end{aligned}
$$

In view of $(\mathcal{H})$, this bilinear form defines a scalar product over $Y$. Moreover, endowed to this scalar product, we easily obtain that $Y$ is a Hilbert space (we refer to [7]). We note the corresponding norm by $\|(y, f)\|_{Y}:=\sqrt{((y, f),(y, f))_{Y}}$. 
Then, for any $\varepsilon>0$, we consider the following extremal problem :

$$
\left(\mathcal{P}_{\varepsilon}\right) \quad\left\{\begin{array}{l}
\inf J_{\varepsilon}(y, f):=\frac{1}{2}\left\|y-y_{o b s}\right\|_{L^{2}\left(q_{T}\right)}^{2}+\frac{\varepsilon}{2}\|f\|_{X}^{2}, \\
\text { subject to } \quad(y, f) \in W
\end{array}\right.
$$

where $W$ is the closed subspace of $Y$ defined by $W:=\{(y, f) \in Y ; L y-f=0$ in $X\}$ and endowed with the norm of $Y$ : precisely, it follows that

$$
\|(y, f)\|_{W}:=\sqrt{\|y\|_{L^{2}\left(q_{T}\right)}^{2}+\varepsilon\|f\|_{X}^{2}}, \quad \forall(y, f) \in W .
$$

The extremal problem $\left(\mathcal{P}_{\varepsilon}\right)$ is well posed : the functional $J_{\varepsilon}$ is continuous over $W$, is strictly convex and is such that $J_{\varepsilon}(y, f) \rightarrow+\infty$ as $\|(y, f)\|_{W} \rightarrow \infty$. Note also that the solution of $\left(\mathcal{P}_{\varepsilon}\right)$ in $W$, depends on $\varepsilon$ but not on $\eta$.

Remark also that if $\varepsilon=0$, then $J_{\varepsilon}$ is a priori only convex leading possibly to distinct minima. This justifies the introduction of the $\varepsilon$-term in the functional $J_{\varepsilon}$. We emphasize however that the $\varepsilon$-term is not a regularization term as it does not improve the regularity of the state $y$.

Eventually, from $(\mathcal{H})$, the solution $\left(y_{\varepsilon}, f_{\varepsilon}\right)$ in $W$ of $\left(\mathcal{P}_{\varepsilon}\right)$ satisfies $\left(y_{\varepsilon}(\cdot, 0), y_{\varepsilon, t}(\cdot, 0)\right) \in \boldsymbol{H}$, so that problem $\left(\mathcal{P}_{\varepsilon}\right)$ is again equivalent to the minimisation of $J_{\varepsilon}$ with respect to $\left(y_{0}, y_{1}, f\right) \in \boldsymbol{H} \times X$.

Proceeding as in Section 2, we introduce a Lagrangian multiplier $\lambda_{\varepsilon} \in X^{\prime}$ and the following mixed formulation: find $\left(\left(y_{\varepsilon}, f_{\varepsilon}\right), \lambda_{\varepsilon}\right) \in Y \times X^{\prime}$ solution of

$$
\left\{\begin{aligned}
a_{\varepsilon}\left(\left(y_{\varepsilon}, f_{\varepsilon}\right),(\bar{y}, \bar{f})\right)+b\left((\bar{y}, \bar{f}), \lambda_{\varepsilon}\right) & =l(\bar{y}, \bar{f}), & & \forall(\bar{y}, \bar{f}) \in Y \\
b\left(\left(y_{\varepsilon}, f_{\varepsilon}\right), \bar{\lambda}\right) & =0, & & \forall \bar{\lambda} \in X^{\prime},
\end{aligned}\right.
$$

where

$$
\begin{aligned}
& a_{\varepsilon}: Y \times Y \rightarrow \mathbb{R}, \quad a_{\varepsilon}((y, f),(\bar{y}, \bar{f})):=\iint_{q_{T}} y \bar{y} d x d t+\varepsilon(f, \bar{f})_{X}, \\
& b: Y \times X^{\prime} \rightarrow \mathbb{R}, \quad b((y, f), \lambda):=\int_{0}^{T}\langle\lambda, L y-f\rangle_{H_{0}^{1}(\Omega), H^{-1}(\Omega)} d t, \\
& l: Y \rightarrow \mathbb{R}, \quad l(y, f):=\iint_{q_{T}} y_{o b s} y d x d t .
\end{aligned}
$$

THEOREM 3.1 Under the hypothesis (H), the following hold:

(i) The mixed formulation (29) is well-posed.

(ii) The unique solution $\left(\left(y_{\varepsilon}, f_{\varepsilon}\right), \lambda_{\varepsilon}\right) \in Y \times X^{\prime}$ is the saddle-point of the Lagrangian $\mathcal{L}_{\varepsilon}: Y \times X^{\prime} \rightarrow$ $\mathbb{R}$ defined by

$$
\mathcal{L}_{\varepsilon}((y, f), \lambda):=\frac{1}{2} a_{\varepsilon}((y, f),(y, f))+b((y, f), \lambda)-l(y, f) .
$$

Moreover, the pair $\left(y_{\varepsilon}, f_{\varepsilon}\right)$ solves the extremal problem $\left(\mathcal{P}_{\varepsilon}\right)$.

(iii) The following estimates hold:

$$
\left\|\left(y_{\varepsilon}, f_{\varepsilon}\right)\right\|_{Y}=\left(\left\|y_{\varepsilon}\right\|_{L^{2}\left(q_{T}\right)}^{2}+\varepsilon\left\|f_{\varepsilon}\right\|^{2}\right)^{1 / 2} \leq\left\|y_{o b s}\right\|_{L^{2}\left(q_{T}\right)}
$$

and

$$
\left\|\lambda_{\varepsilon}\right\|_{L^{2}\left(Q_{T}\right)} \leq 2 \sqrt{C_{\Omega, T}+\eta}\left\|y_{o b s}\right\|_{L^{2}\left(q_{T}\right)}
$$

for some constant $C_{\Omega, T}>0$. 
The proof is very closed to the proof of Theorem 2.1. In particular, the obtention of the inf-sup property is obtained by taking, for any $\lambda \in X^{\prime}, f=0$ and $y$ as in (14) so that the inf-sup constant

$$
\delta_{\varepsilon}:=\inf _{\lambda \in X^{\prime}} \sup _{(y, f) \in Y} \frac{b((y, f), \lambda)}{\|(y, f)\|_{Y}\|\lambda\|_{X^{\prime}}}
$$

is bounded by above by $\left(C_{\Omega, T}+\eta\right)^{-1 / 2}$ uniformly with respect to $\varepsilon$.

Remark in particular that the inequality (33) implies that, at the optimality, since $\varepsilon>0$, the equality $\left\|y-y_{o b s}\right\|_{L^{2}\left(q_{T}\right)}=0$ can not hold if $f_{\varepsilon} \neq 0$.

Remark $\mathbf{5}$ We may also prove the inf-sup property using the variable $f$ : for any $\lambda \in X^{\prime}$, we set $y=0$ and $f=\Delta \lambda \in X$. We get

$$
\sup _{(y, f) \in Y} \frac{b((y, f), \lambda)}{\|(y, f)\|_{Y}\|\lambda\|_{X^{\prime}}} \geq \frac{b((0, \Delta \lambda), \lambda)}{\|(0, \Delta \lambda)\|_{Y}\|\lambda\|_{X^{\prime}}}=\frac{1}{\sqrt{\varepsilon+\eta}}
$$

so that $\delta_{\varepsilon} \geq(\varepsilon+\eta)^{-1 / 2}$. Therefore, the estimate

$$
\left\|\lambda_{\varepsilon}\right\|_{X^{\prime}} \leq \frac{2}{\delta_{\varepsilon}}\left\|y_{o b s}\right\|_{L^{2}\left(q_{T}\right)}
$$

implies that

$$
\left\|\lambda_{\varepsilon}\right\|_{X^{\prime}} \leq 2 \sqrt{\varepsilon+\eta}\left\|y_{o b s}\right\|_{L^{2}\left(q_{T}\right)} .
$$

This argument is valid if and only if $f$ is distributed everywhere in $Q_{T}$.

Remark 6 The estimate (36) implies that the multiplier $\lambda_{\varepsilon}$ vanishes in $X^{\prime}$ as $\varepsilon+\eta \rightarrow 0^{+}$(recall that $\varepsilon$ and $\eta$ can be chosen arbitrarily small in (4)).

\section{Remark 7}

(a) Assuming enough regularity on the solution $\lambda_{\varepsilon}$, precisely that $L \lambda_{\varepsilon} \in L^{2}\left(Q_{T}\right)$ and $\left(\lambda, \lambda_{t}\right)_{t=0, T} \in$ $H_{0}^{1}(\Omega) \times L^{2}(\Omega)$, we easily check that the multiplier $\lambda_{\varepsilon}$ satisfies the following relations :

$$
\left\{\begin{array}{l}
L \lambda_{\varepsilon}=-\left(y_{\varepsilon}-y_{o b s}\right)_{1 \omega}, \quad L y_{\varepsilon}-f_{\varepsilon}=0, \quad \varepsilon f_{\varepsilon}+\Delta \lambda_{\varepsilon}=0 \quad \text { in } Q_{T}, \\
\lambda_{\varepsilon}=0 \quad \text { in } \quad \Sigma_{T}, \\
\lambda_{\varepsilon}=\lambda_{\varepsilon, t}=0 \quad \text { on } \Omega \times\{0, T\} .
\end{array}\right.
$$

Therefore, $\lambda_{\varepsilon}$ is an exact controlled solution of the wave equation through the control $-\left(y_{\varepsilon}-\right.$ $\left.y_{o b s}\right) 1_{\omega}$ and from (36) implies that

$$
\left\|y_{\varepsilon}-y_{o b s}\right\|_{L^{2}\left(q_{T}\right)} \rightarrow 0 \quad \text { as } \quad \varepsilon \rightarrow 0^{+} .
$$

Remark however that $f_{\varepsilon}$ may not be bounded in $X^{\prime}$ uniformly w.r.t. $\varepsilon$ (contrarily to the sequence $\left.\left(\sqrt{\varepsilon} f_{\varepsilon}\right)_{\varepsilon>0}\right)$.

(b) The equality $L y_{\varepsilon}=f_{\varepsilon}$ becomes $\varepsilon L y_{\varepsilon}=-\Delta \lambda_{\varepsilon}$ and leads to $L\left(\varepsilon \Delta^{-1} L y_{\varepsilon}\right)=-L \lambda_{\varepsilon}=\left(y_{\varepsilon}-\right.$ $\left.y_{o b s}\right)_{1_{\omega}}$. Finally, $y_{\varepsilon}$ solves, at least in $\mathcal{D}^{\prime}$, the boundary value problem

$$
\left\{\begin{array}{l}
L\left(\varepsilon\left(-\Delta^{-1}\right) L y_{\varepsilon}\right)+y_{\varepsilon} 1_{\omega}=y_{o b s} 1_{\omega}, \quad \text { in } \quad Q_{T} \\
\left(\varepsilon L y_{\varepsilon}\right)=\left(\varepsilon L y_{\varepsilon}\right)_{t}=0, \quad \text { in } \Omega \times\{0, T\} \\
y_{\varepsilon}=0, \quad \text { on } \quad \Sigma_{T}
\end{array}\right.
$$

or equivalently the variational formulation: find $y_{\varepsilon} \in Z$ (see (3) solution of

$$
\varepsilon \int_{0}^{T}\left\langle L y_{\varepsilon}, L \bar{y}\right\rangle_{H^{-1}(\Omega)} d t+\iint_{q_{T}} y_{\varepsilon} \bar{y} d x d t=\iint_{q_{T}} y_{o b s} \bar{y} d x d t, \quad \forall \bar{y} \in Z
$$


which actually can be obtained directly from the cost $J_{\varepsilon}$, replacing from the beginning $f$ by the term Ly. From the Lax-Milgram lemma, (38) is well-posed and the following estimates hold :

$$
\left\|y_{\varepsilon}\right\|_{L^{2}\left(q_{T}\right)} \leq\left\|y_{o b s}\right\|_{L^{2}\left(q_{T}\right)}, \quad \sqrt{\varepsilon}\left\|L y_{\varepsilon}\right\|_{X} \leq\left\|y_{o b s}\right\|_{L^{2}\left(q_{T}\right)} .
$$

This kind of variational formulation involving the fourth order term Ly $y_{\varepsilon} y$ has been derived and used in [10] in a controllability context.

For any $\varepsilon>0$ and any $y_{o b s} \in L^{2}\left(q_{T}\right)$, the method allows to recover a couple $\left(y_{\varepsilon}, f_{\varepsilon}\right)$ such that $L y_{\varepsilon}=f_{\varepsilon}$ in $Q_{T}$ and $y_{\varepsilon}$ is closed to $y_{o b s}$ (see (37)). In view of the loss of uniqueness, we have no information on the limit of the sequence as $\varepsilon \rightarrow 0$ : the sequence may be unbounded at the limit in $L^{2}\left(Q_{T}\right) \times L^{2}\left(Q_{T}\right)$ even if $y_{o b s}$ is the restriction to $q_{T}$ of a solution of $(1)$.

Remark 8 Contrarily to the inf-sup property, the coercivity of $a_{\varepsilon}$ over $\mathcal{N}(b)$ does not hold uniformly with respect to $\varepsilon$. Recall that the $\varepsilon$-term has been introduced to get a norm for $Y$. This enforces us to add this term in the mixed formulation.

Remark 9 A fortiori, if the initial condition $\left(y_{0}, y_{1}\right) \in \boldsymbol{H}$ is known, one may recover the pair $(y, f) \in Y$ from $y_{o b s}$ and $\left(y_{0}, y_{1}\right)$. The procedure is similar; it suffices to define two additional Lagrange multipliers $\left(\lambda_{1}, \lambda_{2}\right) \in L^{2}(\Omega) \times H_{0}^{1}(\Omega)$ to deal with the constraint $y(\cdot, 0)=y_{0}$ and $y_{t}(\cdot, 0)=$ $y_{1}$ respectively. The extremal problem is now :

$$
\inf _{(y, f) \in W} J_{\varepsilon}(y, f):=\frac{1}{2}\left\|y-y_{o b s}\right\|_{L^{2}\left(q_{T}\right)}^{2}+\frac{\varepsilon}{2}\|f\|_{X^{\prime}}^{2}
$$

where $W$ is the closed subspace of $Y$ defined by

$$
W:=\left\{(y, f) \in Y ; L y-f=0 \text { in } X^{\prime},\left(y(\cdot, 0), y_{t}(\cdot, 0)\right)=\left(y_{0}, y_{1}\right) \text { in } \boldsymbol{H}\right\}
$$

The corresponding mixed formulation is : find $\left(\left(y_{\varepsilon}, f_{\varepsilon}\right),\left(\lambda_{\varepsilon}, \lambda_{\varepsilon, 1}, \lambda_{\varepsilon, 2}\right)\right) \in Y \times \Lambda$ solution of

$$
\left\{\begin{aligned}
a_{\varepsilon}\left(\left(y_{\varepsilon}, f_{\varepsilon}\right),(\bar{y}, \bar{f})\right)+b\left((\bar{y}, \bar{f}),\left(\lambda_{\varepsilon}, \lambda_{\varepsilon, 1}, \lambda_{\varepsilon, 2}\right)\right) & =l_{1}(\bar{y}, \bar{f}), & & \forall(\bar{y}, \bar{f}) \in Y \\
b\left(\left(y_{\varepsilon}, f_{\varepsilon}\right),\left(\bar{\lambda}, \overline{\lambda_{1}}, \overline{\lambda_{2}}\right)\right) & =l_{2}\left(\bar{\lambda}, \overline{\lambda_{1}}, \overline{\lambda_{2}}\right), & & \forall\left(\bar{\lambda}, \overline{\lambda_{1}}, \overline{\lambda_{2}}\right) \in \Lambda,
\end{aligned}\right.
$$

where $a_{\varepsilon}$ is given by 30 and

$$
\begin{gathered}
b: Y \times \Lambda \rightarrow \mathbb{R}, \quad b\left((y, f),\left(\lambda, \lambda_{1}, \lambda_{2}\right)\right):=\iint_{Q_{T}} \lambda(L y-f) d x d t \\
\quad+\left\langle y(\cdot, 0), \lambda_{1}\right\rangle_{L^{2}(\Omega)}+\left\langle y_{t}(\cdot, 0), \lambda_{2}\right\rangle_{H^{-1}(\Omega), H_{0}^{1}(\Omega)} \\
l_{1}: Y \rightarrow \mathbb{R}, \quad l_{1}(y, f):=\iint_{q_{T}} y_{o b s} \bar{y} d x d t \\
l_{2}: \Lambda \rightarrow \mathbb{R}, \quad l_{2}\left(\lambda, \lambda_{1}, \lambda_{2}\right):=\left\langle y_{0}, \lambda_{1}\right\rangle_{L^{2}(\Omega)}+\left\langle y_{1}, \lambda_{2}\right\rangle_{H^{-1}(\Omega), H_{0}^{1}(\Omega)}
\end{gathered}
$$

with $\Lambda:=X^{\prime} \times L^{2}(\Omega) \times H_{0}^{1}(\Omega)$. Using the estimate $(\mathcal{H})$, we easily show that this formulation is well-posed.

In view of Remark 7 (a), we may also associate to the mixed formulation 29 a stabilized version, similarly to 19 .

Again, it is very convenient to "augment" the Lagrangian (see [16]) and consider instead the Lagrangian $\mathcal{L}_{\varepsilon, r}$ defined for any $r>0$ by

$$
\begin{aligned}
& \mathcal{L}_{\varepsilon, r}((y, f), \lambda):=\frac{1}{2} a_{\varepsilon, r}((y, f),(y, f))+b(y, \lambda)-l(y, f), \\
& a_{\varepsilon, r}((y, f),(y, f)):=a_{\varepsilon}((y, f),(y, f))+r\|L y-f\|_{X}^{2}
\end{aligned}
$$


Since $a_{\varepsilon}(y, y)=a_{\varepsilon, r}(y, y)$ on $W$, the Lagrangian $\mathcal{L}_{\varepsilon}$ and $\mathcal{L}_{\varepsilon, r}$ share the same saddle-point. The positive number $r$ is an augmentation parameter. Similarly, proceeding as in Section 2.2. we may also associate to the saddle-point problem $\sup _{\lambda \in X^{\prime}} \inf _{(y, f) \in Y} \mathcal{L}_{r, \varepsilon}((y, f), \lambda)$ a dual problem, which again reduces the search of the couple $\left(y_{\varepsilon}, f_{\varepsilon}\right)$, solution of problem $\left(\mathcal{P}_{\varepsilon}\right)$, to the minimization of a elliptic functional in $\lambda_{\varepsilon}$.

Proposition 3.1 For any $r>0$, let $\left(y_{0}, f_{0}\right) \in Y$ be the unique solution of

$$
a_{\varepsilon, r}\left(\left(y_{0}, f_{0}\right),(\bar{y}, \bar{f})\right)=l(\bar{y}, \bar{f}), \quad \forall(\bar{y}, \bar{f}) \in Y
$$

and let $\mathcal{P}_{\varepsilon, r}$ be the strongly elliptic and symmetric operator from $X^{\prime}$ into $X^{\prime}$ defined by $\mathcal{P}_{\varepsilon, r} \lambda:=$ $-\Delta^{-1}(L y-f)$ where $(y, f) \in Y$ is the unique solution to

$$
a_{\varepsilon, r}((y, f),(\bar{y}, \bar{f}))=b((\bar{y}, \bar{f}), \lambda), \quad \forall(\bar{y}, \bar{f}) \in Y .
$$

Then, the following equality holds

$$
\sup _{\lambda \in X^{\prime}} \inf _{(y, f) \in Y} \mathcal{L}_{\varepsilon, r}((y, f), \lambda)=-\inf _{\lambda \in X^{\prime}} J_{\varepsilon, r}^{\star \star}(\lambda) \quad+\mathcal{L}_{\varepsilon, r}\left(\left(y_{0}, f_{0}\right), 0\right) .
$$

where $J_{\varepsilon, r}^{\star \star}: X^{\prime} \rightarrow X^{\prime}$ is the functional defined by

$$
J_{\varepsilon, r}^{\star \star}(\lambda)=\frac{1}{2} \int_{0}^{T}\left(\mathcal{P}_{\varepsilon, r} \lambda, \lambda\right)_{H_{0}^{1}(\Omega)} d t-b\left(\left(y_{0}, f_{0}\right), \lambda\right) .
$$

Compared to the previous section, the additional unknown $f_{\epsilon}$ on the problem guarantees that the term $\left\|y_{\varepsilon}-y_{o b s}\right\|_{L^{2}\left(q_{T}\right)}$ vanishes at the limit in $\varepsilon$, for any $y_{o b s} \in L^{2}\left(q_{T}\right)$, be a restriction of a solution of (1) or not. The situation is different if additional assumption on $f$ enforces the uniqueness of the pair $(y, f)$ (we refer to [25] and the references therein).

\section{Numerical Analysis of the mixed formulations}

\subsection{Numerical approximation of the mixed formulation (7)}

We consider the numerical analysis of the mixed formulation (7), assuming $r>0$. We follow [12, to which we refer for the details.

Let $Z_{h}$ and $\Lambda_{h}$ be two finite dimensional spaces parametrized by the variable $h$ such that $Z_{h} \subset Z, \Lambda_{h} \subset X^{\prime}$ for every $h>0$. Then, we can introduce the following approximated problems: find the $\left(y_{h}, \lambda_{h}\right) \in Z_{h} \times \Lambda_{h}$ solution of

$$
\left\{\begin{aligned}
a_{r}\left(y_{h}, \bar{y}_{h}\right)+b\left(\bar{y}_{h}, \lambda_{h}\right) & =l\left(\bar{y}_{h}\right), & & \forall \bar{y}_{h} \in Z_{h} \\
b\left(y_{h}, \bar{\lambda}_{h}\right) & =0, & & \forall \bar{\lambda}_{h} \in \Lambda_{h} .
\end{aligned}\right.
$$

The well-posedness of this mixed formulation is again a consequence of two properties: the coercivity of the bilinear form $a_{r}$ on the subset

$$
\mathcal{N}_{h}(b)=\left\{y_{h} \in Z_{h} ; b\left(y_{h}, \lambda_{h}\right)=0 \quad \forall \lambda_{h} \in \Lambda_{h}\right\} .
$$

Actually, from the relation $a_{r}(y, y) \geq(r / \eta)\|y\|_{Z}^{2}$ for all $y \in Z$, the form $a_{r}$ is coercive on the full space $Z$, and so a fortiori on $\mathcal{N}_{h}(b) \subset Z_{h} \subset Z$. The second property is a discrete inf-sup condition. We note $\delta_{h}>0$ by

$$
\delta_{h}:=\inf _{\lambda_{h} \in \Lambda_{h}} \sup _{y_{h} \in Z_{h}} \frac{b\left(y_{h}, \lambda_{h}\right)}{\left\|\lambda_{h}\right\|_{X^{\prime}}\left\|y_{h}\right\|_{Z}} .
$$


For any fixed $h$, the spaces $Z_{h}$ and $\Lambda_{h}$ are of finite dimension so that the infimum and supremum in (42) are reached: moreover, from the property of the bilinear form $a_{r}$, it is standard to check that $\delta_{h}$ is strictly positive. Consequently, for any fixed $h>0$, there exists a unique couple $\left(y_{h}, \lambda_{h}\right)$ solution of 41.

We then have the following estimate.

Proposition 4.1 Let $h>0$. Let $(y, \lambda)$ and $\left(y_{h}, \lambda_{h}\right)$ be the solution of (7) and of (41) respectively. Let $\delta_{h}$ the discrete inf-sup constant defined by (42). Then,

$$
\begin{aligned}
& \left\|y-y_{h}\right\|_{Z} \leq 2\left(1+\frac{1}{\sqrt{\eta} \delta_{h}}\right) d\left(y, Z_{h}\right)+\frac{1}{\sqrt{\eta}} d\left(\lambda, \Lambda_{h}\right), \\
& \left\|\lambda-\lambda_{h}\right\|_{X^{\prime}} \leq\left(2+\frac{1}{\sqrt{\eta} \delta_{h}}\right) \frac{1}{\delta_{h}} d\left(y, Z_{h}\right)+\frac{3}{\sqrt{\eta} \delta_{h}} d\left(\lambda, \Lambda_{h}\right)
\end{aligned}
$$

where $d\left(\lambda, \Lambda_{h}\right):=\inf _{\lambda_{h} \in \Lambda_{h}}\left\|\lambda-\lambda_{h}\right\|_{X^{\prime}}$ and

$$
\begin{aligned}
d\left(y, Z_{h}\right) & :=\inf _{y_{h} \in Z_{h}}\left\|y-y_{h}\right\|_{Z} \\
& =\inf _{y_{h} \in Z_{h}}\left(\left\|y-y_{h}\right\|_{L^{2}\left(q_{T}\right)}^{2}+\eta\left\|L\left(y-y_{h}\right)\right\|_{X}^{2}\right)^{1 / 2} .
\end{aligned}
$$

Proof- From the classical theory of approximation of saddle point problems (see [4, Theorem 5.2.2]) we have that

$$
\begin{aligned}
\left\|y-y_{h}\right\|_{Z} \leq & \left(\frac{2\left\|a_{r}\right\|_{(Z \times Z)^{\prime}}}{\alpha_{0}}+\frac{2\left\|a_{r}\right\|_{(Z \times Z)^{\prime}}^{\frac{1}{2}}\|b\|_{\left(Z \times X^{\prime}\right)^{\prime}}}{\alpha_{0}^{\frac{1}{2}} \delta_{h}}\right) d\left(y, Z_{h}\right) \\
& +\frac{\|b\|_{\left(Z \times X^{\prime}\right)^{\prime}}}{\alpha_{0}} d\left(\lambda, \Lambda_{h}\right)
\end{aligned}
$$

and

$$
\begin{aligned}
\left\|\lambda-\lambda_{h}\right\|_{X^{\prime}} \leq & \left(\frac{2\left\|a_{r}\right\|_{(Z \times Z)^{\prime}}^{\frac{3}{2}}}{\alpha_{0}^{\frac{1}{2}} \delta_{h}}+\frac{\left\|a_{r}\right\|_{(Z \times Z)^{\prime}}\|b\|_{\left(Z \times X^{\prime}\right)^{\prime}}}{\delta_{h}^{2}}\right) d\left(y, Z_{h}\right) \\
& +\frac{3\left\|a_{r}\right\|^{\frac{1}{2}}\|b\|_{\left(Z \times X^{\prime}\right)^{\prime}}}{\alpha_{0}^{\frac{1}{2}} \delta_{h}} d\left(\lambda, \Lambda_{h}\right) .
\end{aligned}
$$

Since, $\left\|a_{r}\right\|_{(Z \times Z)^{\prime}}=\alpha_{0}=1 ;\|b\|_{(Z \times \Lambda)^{\prime}}=\frac{1}{\sqrt{\eta}}$, the result follows.

Remark 10 For $r=0$, the discrete mixed formulation (41) is not well-posed over $Z_{h} \times \Lambda_{h}$ because the form $a_{r=0}$ is not coercive over the discrete kernel of $b$ : the equality $b\left(y_{h}, \lambda_{h}\right)=0$ for all $\lambda_{h} \in \Lambda_{h}$ does not imply in general that Ly $y_{h}$ vanishes. Therefore, the term $r\left\|L y_{h}\right\|_{X}^{2}$, which appears in the Lagrangian $\mathcal{L}_{r}$, may be understood as a stabilization term: for any $h>0$, it ensures the uniform coercivity of the form $a_{r}$ and vanishes at the limit in $h$. We also emphasize that this term is not a regularization term as it does not add any regularity on the solution $y_{h}$.

Let $n_{h}=\operatorname{dim} Z_{h}, m_{h}=\operatorname{dim} \Lambda_{h}$ and let the real matrices $A_{r, h} \in \mathbb{R}^{n_{h}, n_{h}}, B_{h} \in \mathbb{R}^{m_{h}, n_{h}}, J_{h} \in$ $\mathbb{R}^{m_{h}, m_{h}}$ and $L_{h} \in \mathbb{R}^{n_{h}}$ be defined by

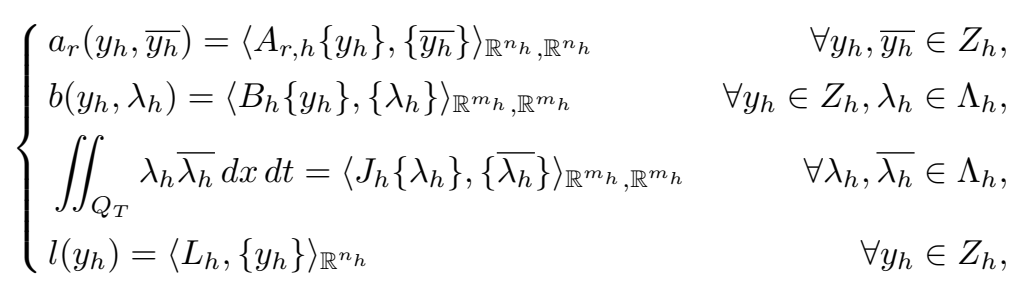


where $\left\{y_{h}\right\} \in \mathbb{R}^{n_{h}}$ denotes the vector associated to $y_{h}$ and $\langle\cdot, \cdot\rangle_{\mathbb{R}^{n_{h}}, \mathbb{R}^{n_{h}}}$ the usual scalar product over $\mathbb{R}^{n_{h}}$. With these notations, the problem (41) reads as follows: find $\left\{y_{h}\right\} \in \mathbb{R}^{n_{h}}$ and $\left\{\lambda_{h}\right\} \in \mathbb{R}^{m_{h}}$ such that

$$
\left(\begin{array}{cc}
A_{r, h} & B_{h}^{T} \\
B_{h} & 0
\end{array}\right)_{\mathbb{R}^{n_{h}+m_{h}, n_{h}+m_{h}}}\left(\begin{array}{l}
\left\{y_{h}\right\} \\
\left\{\lambda_{h}\right\}
\end{array}\right)_{\mathbb{R}^{n_{h}+m_{h}}}=\left(\begin{array}{c}
L_{h} \\
0
\end{array}\right)_{\mathbb{R}^{n_{h}+m_{h}}} .
$$

The matrix $A_{r, h}$ as well as the mass matrix $J_{h}$ are symmetric and positive definite for any $h>0$ and any $r>0$. On the other hand, the matrix of order $m_{h}+n_{h}$ in 48 is symmetric but not positive definite. We use exact integration methods developed in [15] for the evaluation of the coefficients of the matrices. The system $(48)$ is solved using the direct LU decomposition method.

\subsection{1 $C^{1}$-finite elements and order of convergence for $N=1$}

The finite dimensional and conformal space $Z_{h}$ must be chosen such that $L y_{h}$ belongs to $X=$ $L^{2}\left(0, T ; H^{-1}(\Omega)\right)$ for any $y_{h} \in Z_{h}$. This is guaranteed, for instance, as soon as $\varphi_{h}$ possesses second-order derivatives in $L_{l o c}^{2}\left(Q_{T}\right)$. As in [12, we consider a conformal approximation based on functions continuously differentiable with respect to both variables $x$ and $t$.

We introduce a triangulation $\mathcal{T}_{h}$ such that $\overline{Q_{T}}=\cup_{K \in \mathcal{T}_{h}} K$ and we assume that $\left\{\mathcal{T}_{h}\right\}_{h>0}$ is a regular family. We note $h:=\max \left\{\operatorname{diam}(K), K \in \mathcal{T}_{h}\right\}$, where $\operatorname{diam}(K)$ denotes the diameter of $K$. Then, we introduce the space $Z_{h}$ as follows :

$$
Z_{h}=\left\{y_{h} \in Z \subset C^{1}\left(\overline{Q_{T}}\right):\left.z_{h}\right|_{K} \in \mathbb{P}(K) \quad \forall K \in \mathcal{T}_{h}, z_{h}=0 \text { on } \Sigma_{T}\right\},
$$

where $\mathbb{P}(K)$ denotes an appropriate space of functions in $x$ and $t$. In this work, we consider two choices, in the one-dimensional setting (for which $\Omega \subset \mathbb{R}, Q_{T} \subset \mathbb{R}^{2}$ ):

(i) The Bogner-Fox-Schmit (BFS for short) $C^{1}$-element defined for rectangles. It involves 16 degrees of freedom, namely the values of $y_{h}, y_{h, x}, y_{h, t}, y_{h, x t}$ on the four vertices of each rectangle $K$. Therefore $\mathbb{P}(K)=\mathbb{P}_{3, x} \otimes \mathbb{P}_{3, t}$ where $\mathbb{P}_{r, \xi}$ is by definition the space of polynomial functions of order $r$ in the variable $\xi$. We refer to [9, ch. II, sec. 9, p. 94].

(ii) The reduced Hsieh-Clough-Tocher (HCT for short) $C^{1}$-element defined for triangles. This is a so-called composite finite element and involves 9 degrees of freedom, namely, the values of $y_{h}, y_{h, x}, y_{h, t}$ on the three vertices of each triangle $K$. We refer to [9, ch. VII, sec. 46, p. 285] and to [3, 21] where the implementation is discussed.

We also define the finite dimensional space

$$
\Lambda_{h}=\left\{\lambda_{h} \in C^{0}\left(\overline{Q_{T}}\right),\left.\lambda_{h}\right|_{K} \in \mathbb{Q}(K) \quad \forall K \in \mathcal{T}_{h}\right\} .
$$

where $\mathbb{Q}(K)$ denotes the space of affine functions both in $x$ and $t$ on the element $K$.

For any $h>0$, we have $Z_{h} \subset Z$ and $\Lambda_{h} \subset X^{\prime}$.

We then have the following result:

Proposition 4.2 (BFS element for $N=1$ - Rate of Convergence for the norm $Z$ ) Let $h>0$, let $k \in\{1,2\}$ be a positive integer. Let $(y, \lambda)$ and $\left(y_{h}, \lambda_{h}\right)$ be the solution of (7) and (41) respectively. If the solution $(y, \lambda)$ belongs to $H^{k+2}\left(Q_{T}\right) \times H^{k}\left(Q_{T}\right)$, then there exists two positives constants

$$
K_{i}=K_{i}\left(\|y\|_{H^{k+2}\left(Q_{T}\right)},\|c\|_{C^{1}\left(\overline{Q_{T}}\right)},\|d\|_{L^{\infty}\left(Q_{T}\right)}\right), \quad i \in\{1,2\},
$$

independent of $h$, such that

$$
\begin{gathered}
\left\|y-y_{h}\right\|_{Z} \leq K_{1} \frac{h^{k-1}}{\sqrt{\eta}}\left(\left(\sqrt{\eta}+\frac{1}{\delta_{h}}\right)\left(h^{3}+\sqrt{\eta} h\right)+1\right), \\
\left\|\lambda-\lambda_{h}\right\|_{X^{\prime}} \leq K_{2} \frac{h^{k-1}}{\sqrt{\eta} \delta_{h}}\left(\left(\sqrt{\eta}+\frac{1}{\delta_{h}}\right)\left(h^{3}+\sqrt{n} h\right)+1\right) .
\end{gathered}
$$


Proof - From [9, ch. III, sec. 17], for any $\lambda \in H^{k}\left(Q_{T}\right), k \leq 2$, there exists $C_{1}=C_{1}\left(\|\lambda\|_{H^{k}\left(Q_{T}\right)}\right)$ such that

$$
\left\|\lambda-\Pi_{\Lambda_{h}, \mathcal{T}_{h}}(\lambda)\right\|_{X^{\prime}} \leq C_{1} h^{k-1}, \quad \forall h>0
$$

where $\Pi_{\Lambda_{h}, \mathcal{T}_{h}}$ designates the interpolant operator from $X^{\prime}$ to $\Lambda_{h}$ associated to the regular mesh $\mathcal{T}_{h}$. Similarly, for any $y \in H^{k+2}\left(Q_{T}\right)$, there exist $C_{2}=C_{2}\left(\|y\|_{H^{k+2}\left(Q_{T}\right)}\right)$ such that for every $h>0$ we have

$$
\left\|y-\Pi_{Z_{h}, \mathcal{T}_{h}}(y)\right\|_{L^{2}\left(Q_{T}\right)} \leq C_{2} h^{k+2}, \quad\left\|y-\Pi_{Z_{h}, \mathcal{T}_{h}}(y)\right\|_{H^{2}\left(Q_{T}\right)} \leq C_{2} h^{k} .
$$

Then, observing that

$$
\left\|L y-L y_{h}\right\|_{X} \leq K\left(\|c\|_{C^{1}\left(\overline{Q_{T}}\right)},\|d\|_{L^{\infty}\left(Q_{T}\right)}\right)\left\|y-y_{h}\right\|_{H^{2}\left(Q_{T}\right)},
$$

for some positive constant $K$, we get that

$$
\begin{aligned}
d\left(y, Z_{h}\right) & =\inf _{y_{h} \in Z_{h}}\left(\left\|y-y_{h}\right\|_{L^{2}\left(q_{T}\right)}^{2}+\eta\left\|L y-L y_{h}\right\|_{X}^{2}\right)^{2} \\
& \leq C_{2}\left(\left(h^{k+2}\right)^{2}+\eta K^{2}\left(h^{k}\right)^{2}\right)^{1 / 2} \\
& \leq C_{2}\left(h^{k+2}+\sqrt{n} K h^{k}\right)
\end{aligned}
$$

and then from Proposition 4.1, we get that

$$
\left\|y-y_{h}\right\|_{Z} \leq 2\left(1+\frac{1}{\sqrt{\eta} \delta_{h}}\right) C_{2}\left(h^{k+2}+\sqrt{n} K h^{k}\right)+\frac{1}{\sqrt{\eta}} C_{1} h^{k-1} .
$$

Similarly,

$$
\left\|\lambda-\lambda_{h}\right\|_{X^{\prime}} \leq\left(2+\frac{1}{\sqrt{\eta} \delta_{h}}\right) \frac{1}{\delta_{h}} C_{2}\left(h^{k+2}+\sqrt{n} K h^{k}\right)+\frac{3}{\sqrt{\eta} \delta_{h}} C_{1} h^{k-1} .
$$

From the last two estimates, we obtain the conclusion of the proposition.

It remains now to deduce the convergence of the approximated solution $y_{h}$ for the $L^{2}\left(Q_{T}\right)$ norm: this is done using the observability estimate $(\mathcal{H})$. Precisely, we write that $\left(y-y_{h}\right)$ solves

$$
\left\{\begin{array}{l}
L\left(y-y_{h}\right)=-L y_{h} \quad \text { in } Q_{T} \\
\left(\left(y-y_{h}\right),\left(y-y_{h}\right)_{t}\right)(0) \in \boldsymbol{H} \\
y-y_{h}=0 \text { on } \Sigma_{T} .
\end{array}\right.
$$

Therefore using (6), there exists a constant $C\left(C_{\Omega, T}, C_{o b s}\right)$ such that

$$
\left\|y-y_{h}\right\|_{L^{2}\left(Q_{T}\right)}^{2} \leq C\left(C_{\Omega, T}, C_{o b s}\right)\left(\left\|y-y_{h}\right\|_{L^{2}\left(q_{T}\right)}^{2}+\left\|L y_{h}\right\|_{X}^{2}\right)
$$

from which we deduce, in view of the definition of the norm $Y$, that

$$
\left\|y-y_{h}\right\|_{L^{2}\left(Q_{T}\right)} \leq C\left(C_{\Omega, T}, C_{o b s}\right) \max \left(1, \frac{2}{\sqrt{\eta}}\right)\left\|y-y_{h}\right\|_{Z} .
$$

Eventually, by coupling (57) and Proposition 4.2 , we obtain the following result :

Theorem 4.1 (BFS Element for $N=1$ - RAte of CONVERGence For the Norm $L^{2}\left(Q_{T}\right)$ ) Assume that the hypothesis (H) holds. Let $h>0$, let $k \in\{1,2\}$ be a positive integer and let $\eta<1$. Let $(y, \lambda)$ and $\left(y_{h}, \lambda_{h}\right)$ be the solution of (7) and (41) respectively. If the solution $(y, \lambda)$ belongs to $H^{k+2}\left(Q_{T}\right) \times H^{k}\left(Q_{T}\right)$, then there exists two positives constant $K=K\left(\|y\|_{H^{k+2}\left(Q_{T}\right)},\|c\|_{C^{1}\left(\overline{Q_{T}}\right)}\right.$, $\left.\|d\|_{L^{\infty}\left(Q_{T}\right)}, C_{\Omega, T}, C_{o b s}\right)$, independent of $h$, such that

$$
\left\|y-y_{h}\right\|_{L^{2}\left(Q_{T}\right)} \leq K \max \left(1, \frac{2}{\sqrt{\eta}}\right) \frac{h^{k-1}}{\sqrt{\eta}}\left(\left(\sqrt{\eta}+\frac{1}{\delta_{h}}\right)\left(h^{3}+\sqrt{\eta} h\right)+1\right) .
$$


Remark 11 Estimate (58) is not fully satisfactory as it depends on the constant $\delta_{h}$. In view of the complexity of both the constraint Ly $=0$ and of the structure of the space $Z_{h}$, the theoretical estimation of the constant $\delta_{h}$ with respect to $h$ is a difficult problem. However, as discussed at length in [12, Section 2.1], $\delta_{h}$ can be evaluated numerically for any $h$, as the solution of the following generalized eigenvalue problem (taking $\eta=r$, so that $a_{r}(y, y)$ is exactly $\|y\|_{Z}^{2}$ ):

$$
\delta_{h}=\inf \left\{\sqrt{\delta}: B_{h} A_{r, h}^{-1} B_{h}^{T}\left\{\lambda_{h}\right\}=\delta J_{h}\left\{\lambda_{h}\right\}, \quad \forall\left\{\lambda_{h}\right\} \in \mathbb{R}^{m_{h}} \backslash\{0\}\right\}
$$

where the matrix $A_{r, h}, B_{h}$ and $J_{h}$ are defined in (47).

Table 1 reports the values of $\delta_{h}$ for $r=1$ and $r=h^{-2}$ for several values of $h, T=2$, $\omega=(0.1,0.3)$ and the BFS element. As in [12] where the boundary situation is considered with more details, these values suggests that, asymptotically in $h$, the constant $\delta_{r, h}$ behaves like:

$$
\delta_{r, h} \approx C_{r} \frac{1}{\sqrt{r}} \quad \text { as } \quad h \rightarrow 0^{+}
$$

with $C_{r}>0$, a uniformly bounded constant w.r.t. $h$.

\begin{tabular}{|c|cccc|}
\hline$h$ & $7.01 \times 10^{-2}$ & $3.53 \times 10^{-2}$ & $1.76 \times 10^{-2}$ & $8.83 \times 10^{-3}$ \\
\hline$r=1$ & 3.58 & 3.48 & 3.42 & 3.40 \\
$r=h^{-2}$ & $2.53 \times 10^{-1}$ & $1.23 \times 10^{-1}$ & $6.05 \times 10^{-2}$ & $3.01 \times 10^{-2}$ \\
\hline
\end{tabular}

Table 1: $\varepsilon=0: T=2-\delta_{r, h}$ for $r=1$ and $r=h^{-2}$ with respect to $h$.

Consequently, in view of 60 , the right hand side of the estimate (58) of $\left\|y-y_{h}\right\|_{L^{2}\left(Q_{T}\right)}$ behaves, taking $\eta=r$ and $r>1$ so that $\max \left(1, \frac{1}{\sqrt{r}}\right)=1$, like

$$
\left\|y-y_{h}\right\|_{L^{2}\left(Q_{T}\right)} \leq K h^{k-1}\left(\sqrt{r} h+\frac{1}{\sqrt{r}}\right)
$$

and reaches its minimum for $r=1 / h$, leading to $\left\|y-y_{h}\right\|_{L^{2}\left(Q_{T}\right)} \leq K h^{k-1 / 2}$.

Eventually, when the space $Z_{h}$ is based on the HCT element, Theorem 4.1 and Remark 11 still hold for $k=1$. From [9, ch. VII, sec. 48, p. 295], we use that, for $k \in\{0,1\}$, there exists a constant $C_{2}>0$ such

$$
\left\|y-\Pi_{Z_{h}, \mathcal{T}_{h}}(y)\right\|_{L^{2}\left(Q_{T}\right)} \leq C_{2} h^{k+2}, \quad\left\|y-\Pi_{Z_{h}, \mathcal{T}_{h}}(y)\right\|_{H^{2}\left(Q_{T}\right)} \leq C_{2} h^{k} .
$$

Then, we use that the error $\left\|y-y_{h}\right\|_{L^{2}\left(Q_{T}\right)}$ is again controlled by the error on the Lagrange multiplier $\lambda$ through the term $d\left(\lambda, \Lambda_{h}\right)$ in 43 to conclude.

\subsection{Numerical approximation of the mixed formulation (19)}

We address the numerical approximation of the stabilized mixed formulation $(19)$ with $\alpha \in(0,1)$ and $r>0$. Let $h$ be a real parameter. Let $Z_{h}$ and $\widetilde{\Lambda}_{h}$ be two finite dimensional spaces such that

$$
Z_{h} \subset Z, \quad \widetilde{\Lambda}_{h} \subset \Lambda, \quad \forall h>0 .
$$

The problem 19 becomes : find $\left(y_{h}, \lambda_{h}\right) \in Z_{h} \times \widetilde{\Lambda}_{h}$ solution of

$$
\left\{\begin{aligned}
a_{r, \alpha}\left(y_{h}, \bar{y}_{h}\right)+b_{\alpha}\left(\lambda_{h}, \bar{y}_{h}\right) & =l_{1, \alpha}\left(\bar{y}_{h}\right), & & \forall \bar{y}_{h} \in Z_{h} \\
b_{\alpha}\left(\bar{\lambda}_{h}, y_{h}\right)-c_{\alpha}\left(\lambda_{h}, \bar{\lambda}_{h}\right) & =l_{2, \alpha}\left(\bar{\lambda}_{h}\right), & & \forall \bar{\lambda}_{h} \in \widetilde{\Lambda}_{h},
\end{aligned}\right.
$$

Proceeding as in the proof of [4, Theorem 5.5.2], we first easily show that the following estimate holds . 
Lemma 4.1 Let $(y, \lambda) \in Y \times \Lambda$ be the solution of 19$)$ and $\left(y_{h}, \lambda_{h}\right) \in Z_{h} \times \widetilde{\Lambda_{h}}$ be the solution of 62.). Then we have,

$$
\begin{aligned}
\frac{1}{4} \theta_{1}\left\|y-y_{h}\right\|_{Z}^{2}+\frac{1}{4} \theta_{2}\left\|\lambda-\lambda_{h}\right\|_{\widetilde{\Lambda}}^{2} \leq & \left(\frac{\left\|a_{r, \alpha}\right\|^{2}}{\alpha_{a}}+\frac{\left\|b_{\alpha}\right\|^{2}}{\alpha_{c}}+\frac{\theta_{1}}{2}\right) \inf _{\bar{y}_{h} \in Z_{h}}\left\|\bar{y}_{h}-y\right\|_{Z}^{2} \\
& +\left(\frac{\left\|b_{\alpha}\right\|^{2}}{\theta_{1}}+\frac{\alpha^{2}}{\theta_{2}}+\frac{\theta_{2}}{2}\right) \inf _{\bar{\lambda}_{h} \in \widetilde{\Lambda}_{h}}\left\|\bar{\lambda}_{h}-\lambda\right\|_{\Lambda}^{2}
\end{aligned}
$$

with $\left\|a_{r, \alpha}\right\| \leq \max \left(1-\alpha, \eta^{-1} r\right),\left\|b_{\alpha}\right\| \leq \max \left(\eta^{-1 / 2}, \alpha\right)$. Parameters $\theta_{1}$ and $\theta_{2}$ are defined in 20).

Concerning the space $\widetilde{\Lambda}_{h}$, since $L \lambda_{h}$ should belong to $L^{2}\left(Q_{T}\right)$, a natural choice is

$$
\widetilde{\Lambda}_{h}=\left\{\lambda \in Z_{h} ; \lambda(\cdot, 0)=\lambda_{t}(\cdot, 0)=0\right\} .
$$

where $Z_{h} \subset Z$ is defined by (49). Then, using Lemma 4.1 and the estimate (55), we obtain the following result.

Proposition 4.3 (BFS element for $N=1$ - Rate of Convergence - Stabilized formulation) Let $h>0$, let $k \leq 2$ be a positive integer and let $\alpha \in(0,1)$. Let $(y, \lambda)$ and $\left(y_{h}, \lambda_{h}\right)$ be the solution of 19) and 62) respectively. If $(y, \lambda)$ belongs to $H^{k+2}\left(Q_{T}\right) \times H^{k+2}\left(Q_{T}\right)$, then there exists a positive constant $K=K\left(\|y\|_{H^{k+2}\left(Q_{T}\right)},\|c\|_{C^{1}\left(\overline{Q_{T}}\right)},\|d\|_{L^{\infty}\left(Q_{T}\right)}, \alpha, r, \eta\right)$ independent of $h$, such that

$$
\left\|y-y_{h}\right\|_{Z}+\left\|\lambda-\lambda_{h}\right\|_{\Lambda} \leq K h^{k} .
$$

In particular, arguing as in the previous section, we get

Theorem 4.2 (RAte of Convergence for the norm $L^{2}\left(Q_{T}\right)$ Stabilized formulation) Assume that the hypothesis $(\mathcal{H})$ holds. Let $h>0$, let an integer $k \leq 2$. Let $(y, \lambda)$ and $\left(y_{h}, \lambda_{h}\right)$ be the solution of (19) and (62) respectively. If the solution $(y, \lambda)$ belongs to $H^{k+2}\left(Q_{T}\right) \times H^{k+2}\left(Q_{T}\right)$, then there exist a positive constant $K=K\left(\|y\|_{H^{k+2}\left(Q_{T}\right)},\|\lambda\|_{H^{k+2}\left(Q_{T}\right)},\|c\|_{C^{1}\left(\overline{Q_{T}}\right)},\|d\|_{L^{\infty}\left(Q_{T}\right)}, \alpha, r, \eta\right)$ independent of $h$ such that

$$
\left\|y-y_{h}\right\|_{L^{2}\left(Q_{T}\right)} \leq K \frac{h^{k}}{\sqrt{\eta}}
$$

\section{$5 \quad$ Numerical experiments}

We now report and discuss some numerical experiments corresponding to mixed formulation 41 and 62 for $N=1$ and $N=2$.

\subsection{One dimensional case $(N=1)$}

We take $\Omega=(0,1)$. In order to check the convergence of the method, we consider explicit solutions of (1). We define the smooth initial condition (see []):

$$
\left(\mathbf { E X 1 ) } \left\{\begin{array}{l}
y_{0}(x)=16 x^{2}(1-x)^{2}, \\
y_{1}(x)=\left(3 x-4 x^{3}\right) 1_{(0,0.5)}(x)+\left(4 x^{3}-12 x^{2}+9 x-1\right) 1_{(0.5,1)}(x),
\end{array} x \in(0,1)\right.\right.
$$

and $f=0$. The corresponding solution of (1) with $c \equiv 1, d \equiv 0$ is given by

$$
y(x, t)=\sum_{k>0}\left(a_{k} \cos (k \pi t)+\frac{b_{k}}{k \pi} \sin (k \pi t)\right) \sqrt{2} \sin (k \pi x)
$$

with

$$
a_{k}=\frac{32 \sqrt{2}\left(\pi^{2} k^{2}-12\right)}{\pi^{5} k^{5}}\left((-1)^{k}-1\right), \quad b_{k}=\frac{48 \sqrt{2} \sin (\pi k / 2)}{\pi^{4} k^{4}}, \quad k>0 .
$$


We also define the initial data in $H_{0}^{1}(\Omega) \times L^{2}(\Omega)$

$$
\text { (EX2) } \quad y_{0}(x)=1-|2 x-1|, \quad y_{1}(x)=1_{(1 / 3,2 / 3)}(x), \quad x \in(0,1)
$$

for which the Fourier coefficients are

$$
a_{k}=\frac{4 \sqrt{2}}{\pi^{2} k^{2}} \sin (\pi k / 2), \quad b_{k}=\frac{1}{\pi k}(\cos (\pi k / 3)-\cos (2 \pi k / 3)), \quad k>0 .
$$

\subsubsection{The cylindrical case: $q_{T}=\omega \times(0, T)$}

We consider the case $\varepsilon=0$ described in Section 2. We take $\omega=(0.1,0.3)$ and $T=2$ for which the inequality $(\mathcal{H})$ holds true. We consider the BFS finite element with uniform triangulation (each element $K$ of the triangulation $\mathcal{T}_{h}$ is a rectangle of lengths $\Delta x$ and $\Delta t$ so that $h=\sqrt{(\Delta x)^{2}+(\Delta t)^{2}}$. We recall that the direct method amounts to solve, for any $h$, the linear system (48). We use the LU decomposition method. Table 2 collects some norms with respect to $h$ for the initial data (EX1) for $r=1$ and for $\Delta x=\Delta t$. We observe a linear convergence for the variables $y_{h}, \lambda_{h}$ for the $L^{2}$-norm:

$$
\frac{\left\|y-y_{h}\right\|_{L^{2}\left(Q_{T}\right)}}{\|y\|_{L^{2}\left(Q_{T}\right)}}=\mathcal{O}\left(h^{1.03}\right), \quad \frac{\left\|y-y_{h}\right\|_{L^{2}\left(q_{T}\right)}}{\|y\|_{L^{2}\left(q_{T}\right)}}=\mathcal{O}\left(h^{0.98}\right), \quad\left\|\lambda_{h}\right\|_{L^{2}\left(Q_{T}\right)}=\mathcal{O}\left(h^{0.98}\right) .
$$

In agreement with Remark 1, since $y_{o b s}$ is by construction the restriction to $q_{T}$ of a solution of (1), the sequence $\lambda_{h}$, approximation of $\lambda$, vanishes as $h \rightarrow 0$. The $L^{2}$-norm of $L y_{h}$ do also converges to 0 with $h$, with a lower rate:

$$
\left\|L y_{h}\right\|_{L^{2}\left(Q_{T}\right)}=\mathcal{O}\left(h^{0.71}\right) .
$$

\begin{tabular}{|c|ccccc|}
\hline$h$ & $7.01 \times 10^{-2}$ & $3.53 \times 10^{-2}$ & $1.76 \times 10^{-2}$ & $8.83 \times 10^{-3}$ & $4.42 \times 10^{-3}$ \\
\hline$\frac{\left\|y-y_{h}\right\|_{L^{2}\left(Q_{T}\right)}}{\|y\|_{L^{2}\left(Q_{T}\right)}}$ & $9.55 \times 10^{-2}$ & $4.58 \times 10^{-2}$ & $2.24 \times 10^{-2}$ & $1.10 \times 10^{-2}$ & $5.52 \times 10^{-3}$ \\
$\frac{\left\|y-y_{h}\right\|_{L^{2}\left(q_{T}\right)}}{\|y\|_{L^{2}\left(q_{T}\right)}}$ & $8.35 \times 10^{-2}$ & $4.28 \times 10^{-2}$ & $2.16 \times 10^{-2}$ & $1.09 \times 10^{-2}$ & $5.51 \times 10^{-3}$ \\
$\left\|L y_{h}\right\|_{L^{2}\left(Q_{T}\right)}$ & $5.62 \times 10^{-3}$ & $3.21 \times 10^{-3}$ & $1.78 \times 10^{-3}$ & $9.99 \times 10^{-4}$ & $8.54 \times 10^{-4}$ \\
$\left\|\lambda_{h}\right\|_{L^{2}\left(Q_{T}\right)}$ & $2.67 \times 10^{-5}$ & $1.37 \times 10^{-5}$ & $6.89 \times 10^{-6}$ & $3.44 \times 10^{-6}$ & $1.76 \times 10^{-6}$ \\
$\kappa$ & $1.4 \times 10^{10}$ & $4.6 \times 10^{11}$ & $1.3 \times 10^{13}$ & $4.2 \times 10^{14}$ & $1.3 \times 10^{16}$ \\
$\operatorname{card}\left(\left\{\lambda_{h}\right\}\right)$ & 861 & 3321 & 13041 & 51681 & 205761 \\
$\sharp$ CG iterates & 27 & 42 & 70 & 96 & 90 \\
\hline
\end{tabular}

Table 2: Example EX1 - $r=1-T=2-\|y\|_{L^{2}\left(q_{T}\right)}=5.95 \times 10^{-2}-\|y\|_{L^{2}\left(Q_{T}\right)}=1.59 \times 10^{-1}$.

We also check that the minimization of the functional $J_{r}^{\star \star}$ introduced in Proposition 2.3 leads exactly to the same result: we recall that the minimization of the functional $J_{r}^{\star \star}$ corresponds to the resolution of the associate mixed formulation by an iterative Uzawa type method. The minimization is done using a conjugate gradient algorithm ( we refer to [12, Section 2.2] for the algorithm). Each iteration amounts to solve a linear system involving the matrix $A_{r, h}$ which is sparse, symmetric and positive definite. The Cholesky method is used. The performance of the algorithm depends on the conditioning number of the operator $\mathcal{P}_{r}$ : precisely, it is known that (see for instance [14]),

$$
\left\|\lambda^{n}-\lambda\right\|_{L^{2}\left(Q_{T}\right)} \leq 2 \sqrt{\nu\left(\mathcal{P}_{r}\right)}\left(\frac{\sqrt{\nu\left(\mathcal{P}_{r}\right)}-1}{\sqrt{\nu\left(\mathcal{P}_{r}\right)}+1}\right)^{n}\left\|\lambda^{0}-\lambda\right\|_{L^{2}\left(Q_{T}\right)}, \quad \forall n \geq 1
$$

where $\lambda$ minimizes $J_{r}^{\star \star} . \nu\left(\mathcal{P}_{r}\right)=\left\|\mathcal{P}_{r}\right\|\left\|\mathcal{P}_{r}^{-1}\right\|$ denotes the condition number of the operator $\mathcal{P}_{r}$. As discussed in [12, Section 4.4], the conditioning number of $\mathcal{P}_{r}$ restricted to $\Lambda_{h} \subset L^{2}\left(Q_{T}\right)$ behaves 
asymptotically as $C_{r}^{-2} h^{-2}$. Table 2 reports the number of iterations of the algorithm, initiated with $\lambda^{0}=0$ in $Q_{T}$. We take $\epsilon=10^{-10}$ as a stopping threshold for the algorithm (the algorithm is stopped as soon as the norm of the residue $g^{n}$ given here by $L y^{n}$ satisfies $\left.\left\|g^{n}\right\|_{L^{2}\left(Q_{T}\right)} \leq 10^{-10}\left\|g^{0}\right\|_{L^{2}\left(Q_{T}\right)}\right)$.

Table 2 reports the number of iterates to reach convergence, with respect to $h$. We observe that this number is sub-linear with respect to $h$, precisely, with respect to the dimension $m_{h}=\operatorname{card}\left(\left\{\lambda_{h}\right\}\right)$ of the approximated problems. This renders this method very attractive from a numerical point of view.

From Remark 6, we also check the convergence w.r.t. $h$ when we assume from the beginning that the multiplier $\lambda$ vanishes (see Table 3). This amounts to minimize the functional $J_{r}$ given by (17) or, equivalently, to perform exactly one iteration of the conjugate gradient algorithm we have just discussed. With $r=1$, we observe a weaker convergence :

$$
\frac{\left\|y-y_{h}\right\|_{L^{2}\left(Q_{T}\right)}}{\|y\|_{L^{2}\left(Q_{T}\right)}}=\mathcal{O}\left(h^{0.574}\right), \quad \frac{\left\|y-y_{h}\right\|_{L^{2}\left(q_{T}\right)}}{\|y\|_{L^{2}\left(q_{T}\right)}}=\mathcal{O}\left(h^{0.94}\right) .
$$

This example illustrates that the convergence of $L y_{h}$ to 0 in the norm $L^{2}\left(0, T, H^{-1}(0,1)\right)$ is enough here to guarantee the convergence of the approximation $y_{h}$ : we get that $h\left\|L y_{h}\right\|_{L^{2}\left(Q_{T}\right)} \approx$ $\left\|L y_{h}\right\|_{L^{2}\left(0, T ; H^{-1}(0,1)\right.}=\mathcal{O}\left(h^{0.3}\right)$ while $\left\|L y_{h}\right\|_{L^{2}\left(Q_{T}\right)}$ slightly increases. Obviously, in this specific situation, a larger $r$ (acting as a penalty term) independent of $h$ yields a lower $\left\|L y_{h}\right\|_{L^{2}\left(Q_{T}\right)}$ norm.

\begin{tabular}{|c|ccccc|}
\hline$h$ & $7.01 \times 10^{-2}$ & $3.53 \times 10^{-2}$ & $1.76 \times 10^{-2}$ & $8.83 \times 10^{-3}$ & $4.42 \times 10^{-3}$ \\
\hline$\frac{\left\|y-y_{h}\right\|_{L^{2}\left(Q_{T}\right)}}{\|y\|_{L^{2}\left(Q_{T}\right)}}$ & $9.74 \times 10^{-2}$ & $4.90 \times 10^{-2}$ & $2.84 \times 10^{-2}$ & $2.16 \times 10^{-2}$ & $2.01 \times 10^{-2}$ \\
$\frac{\left\|y-y_{h}\right\|_{L^{2}\left(q_{T}\right)}}{\|y\|_{L^{2}\left(q_{T}\right)}}$ & $8.35 \times 10^{-2}$ & $4.28 \times 10^{-2}$ & $2.18 \times 10^{-2}$ & $1.12 \times 10^{-2}$ & $6.21 \times 10^{-3}$ \\
$\left\|L y_{h}\right\|_{L^{2}\left(Q_{T}\right)}$ & $7.72 \times 10^{-3}$ & $1.11 \times 10^{-2}$ & $2.01 \times 10^{-2}$ & $3.40 \times 10^{-2}$ & $4.79 \times 10^{-2}$ \\
\hline
\end{tabular}

Table 3: Example EX1 $-r=1-T=2-\lambda$ fixed to zero.

On the contrary, we check that the convergence to 0 of $\left\|y-y_{h}\right\|_{L^{2}\left(Q_{T}\right)}$ is lost when the inequality $(\mathcal{H}$ ) is not satisfied: Table 4 collects the norms w.r.t. $h$ for the same data except the value $T=1$ (for which the uniqueness of the solution is lost): we observe that $\left\|y-y_{h}\right\|_{L^{2}\left(Q_{T}\right)}$ increases as $h \rightarrow 0$. As an illustration of the loss of uniqueness, these value also yields to a larger conditioning number $\kappa$ of the matrix $A_{r, h}$.

\begin{tabular}{|c|ccccc|}
\hline$h$ & $7.01 \times 10^{-2}$ & $3.53 \times 10^{-2}$ & $1.76 \times 10^{-2}$ & $8.83 \times 10^{-3}$ & $4.42 \times 10^{-3}$ \\
\hline$\frac{\left\|y-y_{h}\right\|_{L^{2}\left(Q_{T}\right)}}{\|y\|_{L^{2}\left(Q_{T}\right)}}$ & $1.21 \times 10^{-1}$ & $1.08 \times 10^{-1}$ & $1.34 \times 10^{-1}$ & $2.42 \times 10^{-1}$ & $5.19 \times 10^{-1}$ \\
$\frac{\left\|y-y_{h}\right\|_{L^{2}\left(q_{T}\right)}}{\|y\|_{L^{2}\left(q_{T}\right)}}$ & $8.40 \times 10^{-2}$ & $4.34 \times 10^{-2}$ & $2.22 \times 10^{-2}$ & $1.12 \times 10^{-2}$ & $5.62 \times 10^{-3}$ \\
$\left\|L y_{h}\right\|_{L^{2}\left(Q_{T}\right)}$ & $5.62 \times 10^{-2}$ & $2.77 \times 10^{-2}$ & $2.63 \times 10^{-2}$ & $2.25 \times 10^{-2}$ & $2.15 \times 10^{-2}$ \\
$\left\|\lambda_{h}\right\|_{L^{2}\left(Q_{T}\right)}$ & $1.84 \times 10^{-5}$ & $9.48 \times 10^{-6}$ & $4.76 \times 10^{-6}$ & $2.38 \times 10^{-6}$ & $1.19 \times 10^{-6}$ \\
$\kappa$ & $1.2 \times 10^{11}$ & $9.8 \times 10^{12}$ & $1.1 \times 10^{15}$ & $1.5 \times 10^{17}$ & $2.7 \times 10^{19}$ \\
\hline
\end{tabular}

Table 4: Example EX1 - $r=1-T=1-\left\|y_{e x}\right\|_{L^{2}\left(q_{T}\right)}=4.21 \times 10^{-2}-\left\|y_{e x}\right\|_{L^{2}\left(Q_{T}\right)}=1.12 \times 10^{-1}$.

Similar conclusions hold with the less regular initial data (EX2). Numerical results are reported in Table 5. We still observe a linear convergence w.r.t. $h$ of $\left\|y-y_{h}\right\|_{L^{2}\left(Q_{T}\right)},\left\|y-y_{h}\right\|_{L^{2}\left(q_{T}\right)}$ and $\left\|\lambda_{h}\right\|_{L^{2}\left(Q_{T}\right)}$. One notable difference is that the convergence rate is weaker for the norm $\left\|L y_{h}\right\|_{L^{2}\left(Q_{T}\right)}$ :

$$
\left\|L y_{h}\right\|_{L^{2}\left(Q_{T}\right)}=\mathcal{O}\left(h^{0.123}\right) .
$$

Again, this is enough to guarantee the convergence of $y_{h}$ toward a solution of the wave equation: recall that then $\left\|L y_{h}\right\|_{L^{2}\left(0, T ; H^{-1}(0,1)\right)}=\mathcal{O}\left(h^{1.123}\right)$. We also observe that the number of iterates 
in the $C G$ algorithm remains largely sub-linear but is slightly larger: precisely, we have $\sharp$ iter $=\mathcal{O}\left(h^{-0.71}\right)$. Table 6 illustrates the case $T=1$ while Table 7 illustrates the minimization of $J_{r}$ (see 17), both for $r=1$.

\begin{tabular}{|c|ccccc|}
\hline$h$ & $7.01 \times 10^{-2}$ & $3.53 \times 10^{-2}$ & $1.76 \times 10^{-2}$ & $8.83 \times 10^{-3}$ & $4.42 \times 10^{-3}$ \\
\hline$\frac{\left\|y-y_{h}\right\|_{L^{2}\left(Q_{T}\right)}}{\|y\|_{L^{2}\left(Q_{T}\right)}}$ & $1.01 \times 10^{-1}$ & $4.81 \times 10^{-2}$ & $2.34 \times 10^{-2}$ & $1.15 \times 10^{-2}$ & $5.68 \times 10^{-3}$ \\
$\frac{\left\|y-y_{h}\right\|_{L^{2}\left(q_{T}\right)}}{\|y\|_{L^{2}\left(q_{T}\right)}}$ & $1.34 \times 10^{-1}$ & $5.05 \times 10^{-2}$ & $2.37 \times 10^{-2}$ & $1.16 \times 10^{-2}$ & $5.80 \times 10^{-3}$ \\
$\left\|L y_{h}\right\|_{L^{2}\left(Q_{T}\right)}$ & $7.18 \times 10^{-2}$ & $6.59 \times 10^{-2}$ & $6.11 \times 10^{-2}$ & $5.55 \times 10^{-2}$ & $5.10 \times 10^{-2}$ \\
$\left\|\lambda_{h}\right\|_{L^{2}\left(Q_{T}\right)}$ & $1.07 \times 10^{-4}$ & $4.70 \times 10^{-5}$ & $2.32 \times 10^{-5}$ & $1.15 \times 10^{-5}$ & $5.76 \times 10^{-6}$ \\
$\sharp$ CG iterates & 29 & 46 & 83 & 133 & 201 \\
\hline
\end{tabular}

Table 5: Example EX2 - $r=1-T=2-\|y\|_{L^{2}\left(q_{T}\right)}=1.56 \times 10^{-1}-\|y\|_{L^{2}\left(Q_{T}\right)}=4.14 \times 10^{-1}$.

\begin{tabular}{|c|ccccc|}
\hline$h$ & $7.01 \times 10^{-2}$ & $3.53 \times 10^{-2}$ & $1.76 \times 10^{-2}$ & $8.83 \times 10^{-3}$ & $4.42 \times 10^{-3}$ \\
\hline$\frac{\left\|y-y_{h}\right\|_{L^{2}\left(Q_{T}\right)}}{\|y\|_{L^{2}\left(Q_{T}\right)}}$ & $2.74 \times 10^{-1}$ & $4.15 \times 10^{-1}$ & $6.30 \times 10^{-1}$ & 1.21 & 2.62 \\
$\frac{\left\|y-y_{h}\right\|_{L^{2}\left(q_{T}\right)}}{\|y\|_{L^{2}\left(q_{T}\right)}}$ & $1.37 \times 10^{-1}$ & $5.76 \times 10^{-2}$ & $2.89 \times 10^{-2}$ & $2.41 \times 10^{-2}$ & $7.76 \times 10^{-3}$ \\
$\left\|L y_{h}\right\|_{L^{2}\left(Q_{T}\right)}$ & $5.97 \times 10^{-2}$ & $4.96 \times 10^{-2}$ & $4.96 \times 10^{-2}$ & $4.52 \times 10^{-2}$ & $4.21 \times 10^{-2}$ \\
$\left\|\lambda_{h}\right\|_{L^{2}\left(Q_{T}\right)}$ & $4.97 \times 10^{-5}$ & $2.32 \times 10^{-5}$ & $1.15 \times 10^{-5}$ & $5.76 \times 10^{-5}$ & $2.87 \times 10^{-6}$ \\
\hline
\end{tabular}

Table 6: Example EX2 - $r=1-T=1-\|y\|_{L^{2}\left(q_{T}\right)}=1.104 \times 10^{-1}-\|y\|_{L^{2}\left(Q_{T}\right)}=2.93 \times 10^{-1}$.

\begin{tabular}{|c|ccccc|}
\hline$h$ & $7.01 \times 10^{-2}$ & $3.53 \times 10^{-2}$ & $1.76 \times 10^{-2}$ & $8.83 \times 10^{-3}$ & $4.42 \times 10^{-3}$ \\
\hline$\frac{\left\|y-y_{h}\right\|_{L^{2}\left(Q_{T}\right)}}{\|y\|_{L^{2}\left(Q_{T}\right)}}$ & $1.02 \times 10^{-1}$ & $5.27 \times 10^{-2}$ & $3.18 \times 10^{-2}$ & $2.48 \times 10^{-2}$ & $2.25 \times 10^{-2}$ \\
$\frac{\left\|y-y_{h}\right\|_{L^{2}\left(q_{T}\right)}}{\|y\|_{L^{2}\left(q_{T}\right)}}$ & $1.34 \times 10^{-1}$ & $5.06 \times 10^{-2}$ & $2.37 \times 10^{-2}$ & $1.21 \times 10^{-2}$ & $6.65 \times 10^{-3}$ \\
$\left\|L y_{h}\right\|_{L^{2}\left(Q_{T}\right)}$ & $7.43 \times 10^{-2}$ & $7.43 \times 10^{-2}$ & $8.65 \times 10^{-2}$ & $1.10 \times 10^{-1}$ & $1.37 \times 10^{-2}$ \\
\hline
\end{tabular}

Table 7: Example EX2 - $r=1-T=2-\lambda$ fixed to zero.

We end this section with some numerical results for the stabilized mixed formulation (62). The main difference is that the multiplier $\lambda$ is approximated in a much richer space $\widetilde{\Lambda}_{h}$ (see 64) leading to larger linear system. Table 8 consider the case of the example EX2 with $T=2$ and $\alpha=1 / 2$. In order to compare with the formulation (41), we take again $r=1$. We observe the convergence w.r.t $h$ and obtain slightly better rates and constants than in Table 5 in particular, we have $\left\|y-y_{h}\right\|_{L^{2}\left(Q_{T}\right)} /\|y\|_{L^{2}\left(Q_{T}\right)}=\mathcal{O}\left(h^{1.10}\right)$. This is partially due to the fact that the space $\widetilde{\Lambda}_{h}$ used for the variable $\lambda_{h}$ in (62) is richer than the space $\Lambda_{h}$ used in (41). However, for $\alpha=0$ leading to the non stabilized mixed formulation, the space $\widetilde{\Lambda}_{h}$ is too rich and produce poor result, while we obtain very similar results for any values of $\alpha$ in $(0,1]$. Finally, we also check that - in contrast with the mixed formulation (41) - the positive parameter $r$ does not affect the numerical results.

We also emphasize that this variational method which requires a finite element discretization of the time-space $Q_{T}$ is particularly well-adapted to mesh optimization. Still for the example EX2, Figure 1 depicts a sequence of four distinct meshes of $Q_{T}=(0,1) \times(0, T)$ : the sequence is initiated with a coarse and regularly distributed mesh. The three other meshes are successively obtained by local refinement based on the norm of the gradient of $y_{h}$ on each triangle of $\mathcal{T}_{h}$. As expected, the refinement is concentrated around the lines of singularity of $y_{h}$ travelling in $Q_{T}$, generated by the singularity of the initial position $y_{0}$. The four meshes contain 792,2 108,7 902 and 14717 triangles respectively (see Table 9). The results obtained using the reduced HCT finite element are reported in Table 9 . 


\begin{tabular}{|c|ccccc|}
\hline$h$ & $7.01 \times 10^{-2}$ & $3.53 \times 10^{-2}$ & $1.76 \times 10^{-2}$ & $8.83 \times 10^{-3}$ & $4.42 \times 10^{-3}$ \\
\hline$\frac{\left\|y-y_{h}\right\|_{L^{2}\left(Q_{T}\right)}}{\|y\|_{L^{2}\left(Q_{T}\right)}}$ & $8.48 \times 10^{-2}$ & $4.01 \times 10^{-2}$ & $1.85 \times 10^{-2}$ & $8.66 \times 10^{-3}$ & $4.01 \times 10^{-3}$ \\
$\frac{\left\|y-y_{h}\right\|_{L^{2}\left(q_{T}\right)}}{\|y\|_{L^{2}\left(q_{T}\right)}}$ & $2.80 \times 10^{-1}$ & $7.26 \times 10^{-2}$ & $2.61 \times 10^{-2}$ & $1.12 \times 10^{-2}$ & $5.05 \times 10^{-3}$ \\
$\left\|L y_{h}\right\|_{L^{2}\left(Q_{T}\right)}$ & $7.25 \times 10^{-2}$ & $6.59 \times 10^{-2}$ & $6.16 \times 10^{-2}$ & $5.58 \times 10^{-2}$ & $5.08 \times 10^{-2}$ \\
$\left\|\lambda_{h}\right\|_{L^{2}\left(Q_{T}\right)}$ & $4.11 \times 10^{-3}$ & $2.04 \times 10^{-3}$ & $1.49 \times 10^{-3}$ & $1.01 \times 10^{-3}$ & $7.37 \times 10^{-4}$ \\
\hline
\end{tabular}

Table 8: Example EX2 $-r=1-T=2-\alpha=1 / 2-\|y\|_{L^{2}\left(q_{T}\right)}=5.95 \times 10^{-2}-\|y\|_{L^{2}\left(Q_{T}\right)}=$ $1.59 \times 10^{-1}$.
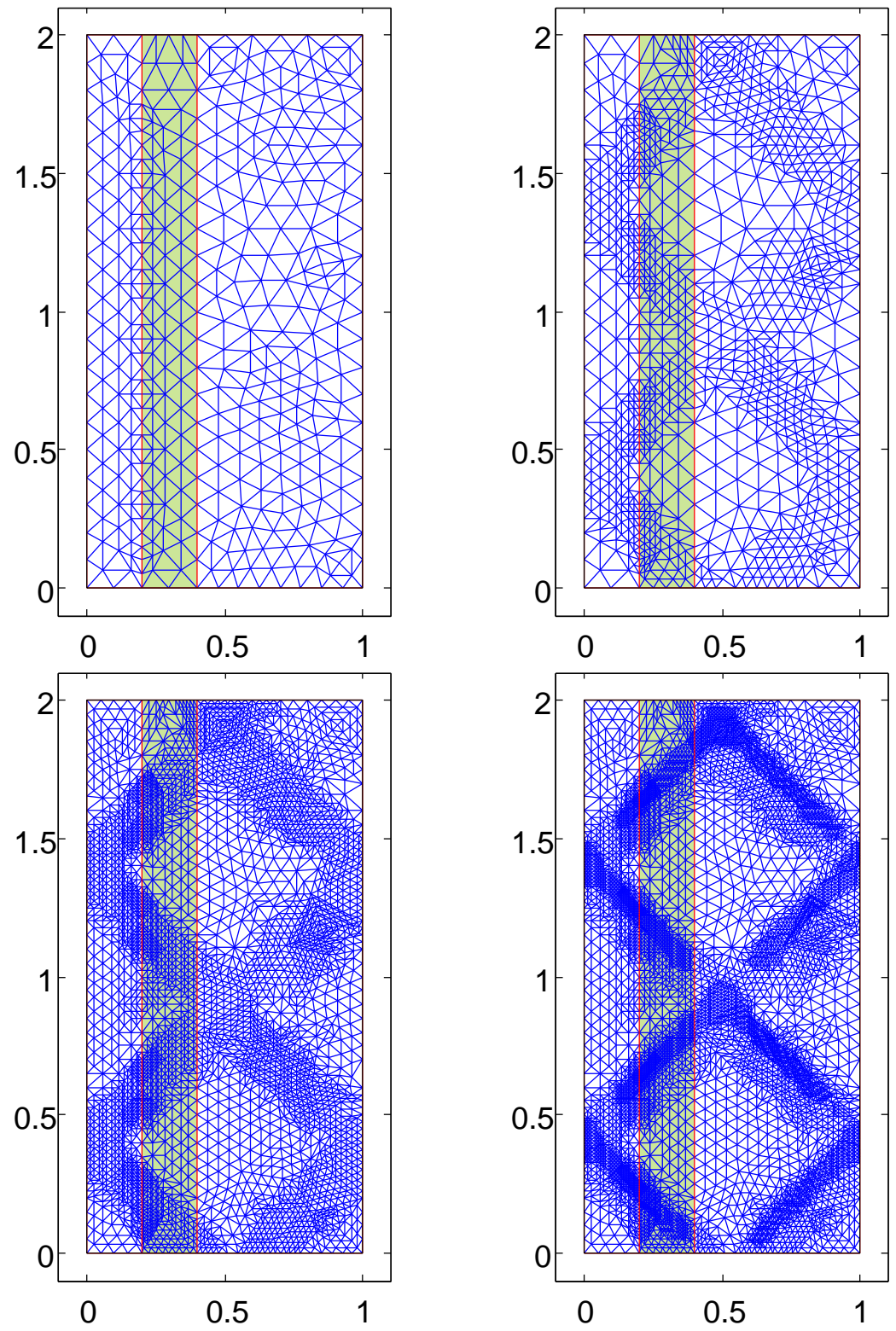

Figure 1: Iterative refinement of the triangular mesh over $Q_{T}$ with respect to the variable $y$. 


\begin{tabular}{|c|cccc|}
\hline Mesh number & 1 & 2 & 3 & 4 \\
\hline$\sharp$ elements & 792 & 2108 & 7902 & 14717 \\
$\sharp$ points & 429 & 1101 & 4041 & 7462 \\
$\frac{\left\|y-y_{h}\right\|_{L^{2}\left(Q_{T}\right)}}{\|y\|_{L^{2}\left(Q_{T}\right)}}$ & $1.34 \times 10^{-2}$ & $8.69 \times 10^{-3}$ & $6.01 \times 10^{-3}$ & $5.9 \times 10^{-3}$ \\
$\|\lambda\|_{L^{2}\left(Q_{T}\right)}$ & $1.14 \times 10^{-5}$ & $7.99 \times 10^{-6}$ & $5.02 \times 10^{-6}$ & $4.79 \times 10^{-6}$ \\
\hline
\end{tabular}

Table 9: Example (EX2) - Information concerning the meshes and approximation errors for mesh adaptation strategy.

\subsubsection{The non-cylindrical case}

We numerically illustrate the reconstruction of the state of the wave equation (1) from measurements $y_{o b s}$ which are available in domains $q_{T} \subset Q_{T}$ non-constant in time (considered recently in [7] in a controllability context). Time dependent domains also appears for time under sampled observations (or measurements): we refer to [11]. In what follows we take $T=2$ and $q_{T}$ to be one of the two following domains:

$$
\begin{aligned}
q_{T}^{1}:=\{(x, t) & \left.\in Q_{T} \text { such that }\left|x-\frac{3 t}{5 T}-\frac{1}{5}\right|<\frac{1}{10} \text { for every } t \in(0, T)\right\}, \\
q_{T}^{2}:= & \left(\frac{1}{10}, \frac{2}{10}\right) \times\left(0, \frac{T}{4}\right) \bigcup\left(\frac{1}{2}, \frac{7}{10}\right) \times\left(\frac{T}{4}, \frac{T}{2}\right) \\
& \bigcup\left(\frac{1}{5}, \frac{2}{5}\right) \times\left(\frac{T}{2}, \frac{3 T}{4}\right) \bigcup\left(\frac{7}{10}, \frac{9}{10}\right) \times\left(\frac{3 T}{4}, T\right) .
\end{aligned}
$$

These two pairs $\left(T, q_{T}^{i}\right) i=1,2$ satisfy the standard geometric optic condition: therefore, using [7, Proposition 2.1, inequality $\mathcal{H}$ holds true. Both domains $q_{T}^{1}$ and $q_{T}^{2}$ are displayed in Figure 2 with the coarsest of the meshes that are used for the numerical experiments in this section.

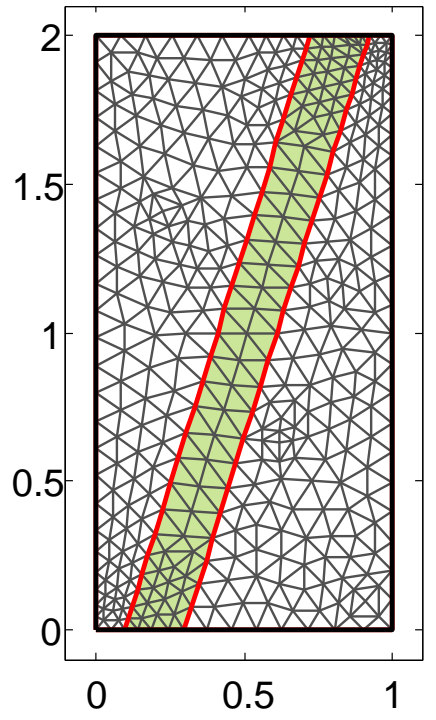

(a)

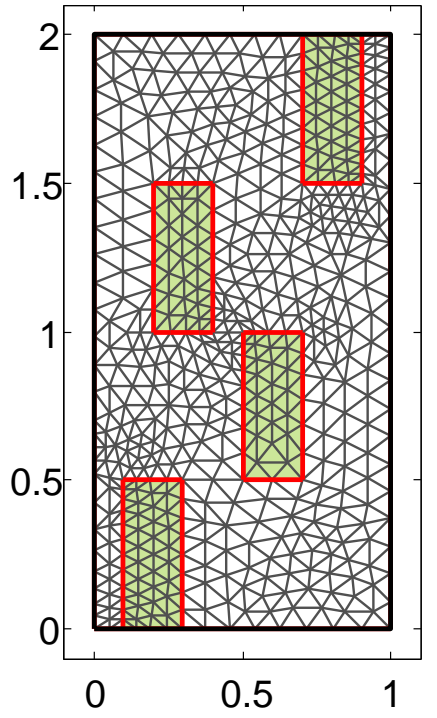

(b)

Figure 2: Domain $q_{T}^{1}$ (a) and domain $q_{T}^{2}$ (b) triangulated using some coarse meshes.

We consider five levels of regular triangular meshes and use the reduced Hsieh-Clough-Tocher finite element. We illustrate our method on the reconstruction of the solution of the wave equation corresponding to initial data (EX2) considered in Section 5.1.1. 
Since domains $q_{T}^{1}$ and $q_{T}^{2}$ satisfy the geometric optic condition, we obtain similar results as in the case $q_{T}=\omega \times(0, T)$ studied in the previous section. More precisely, these results are reported in Table 10 and Table 11 for domain $q_{T}^{1}$ and $q_{T}^{2}$ respectively.

\begin{tabular}{|c|ccccc|}
\hline$h$ & $7.18 \times 10^{-2}$ & $3.59 \times 10^{-2}$ & $1.79 \times 10^{-2}$ & $9 \times 10^{-3}$ & $4.5 \times 10^{-3}$ \\
\hline$\frac{\left\|y-y_{h}\right\|_{L^{2}\left(Q_{T}\right)}}{\|y\|_{L^{2}\left(Q_{T}\right)}}$ & $2.02 \times 10^{-2}$ & $7.83 \times 10^{-3}$ & $3.32 \times 10^{-3}$ & $1.36 \times 10^{-3}$ & $6.27 \times 10^{-4}$ \\
$\frac{\left\|y-y_{h}\right\|_{L^{2}\left(q_{T}\right)}}{\|y\|_{L^{2}\left(q_{T}\right)}}$ & $1.85 \times 10^{-2}$ & $6.69 \times 10^{-3}$ & $2.40 \times 10^{-3}$ & $1.03 \times 10^{-3}$ & $4.56 \times 10^{-4}$ \\
$\left\|L y_{h}\right\|_{L^{2}\left(Q_{T}\right)}$ & 3.41 & 3.78 & 4.15 & 4.47 & 4.76 \\
$\left\|\lambda_{h}\right\|_{L^{2}\left(Q_{T}\right)}$ & $1.97 \times 10^{-5}$ & $7.03 \times 10^{-6}$ & $1.70 \times 10^{-6}$ & $4.14 \times 10^{-7}$ & $1.10 \times 10^{-7}$ \\
$\kappa$ & $1.18 \times 10^{8}$ & $1.84 \times 10^{9}$ & $1.61 \times 10^{10}$ & $1.75 \times 10^{11}$ & $1.38 \times 10^{12}$ \\
$\operatorname{card}\left(\left\{\lambda_{h}\right\}\right)$ & 429 & 1633 & 6369 & 25153 & 99969 \\
$\sharp$ CG iterates & 108 & 206 & 392 & 954 & 2009 \\
\hline
\end{tabular}

Table 10: Observation domain $q_{T}^{1}$. Example EX2 - $r=1-T=2-\|y\|_{L^{2}\left(q_{T}\right)}=2.75 \times 10^{-1}-$ $\|y\|_{L^{2}\left(Q_{T}\right)}=5.87 \times 10^{-1}$.

Remark that the number of iterations needed for the conjugate gradient algorithm in order to achieve a residual smaller than $10^{-10}$ when we minimize the functional $J^{\star \star}$ over $\Lambda_{h}$ is slightly larger than in the situations described in the previous section.

\begin{tabular}{|c|ccccc|}
\hline$h$ & $6.24 \times 10^{-2}$ & $3.12 \times 10^{-2}$ & $1.56 \times 10^{-2}$ & $7.8 \times 10^{-3}$ & $3.9 \times 10^{-3}$ \\
\hline$\frac{\left\|y-y_{h}\right\|_{L^{2}\left(Q_{T}\right)}}{\|y\|_{L^{2}\left(Q_{T}\right)}}$ & $1.38 \times 10^{-2}$ & $6.37 \times 10^{-3}$ & $2.64 \times 10^{-3}$ & $1.15 \times 10^{-3}$ & $5.25 \times 10^{-4}$ \\
$\frac{\left\|y-y_{h}\right\|_{L^{2}\left(q_{T}\right)}}{\|y\|_{L^{2}\left(q_{T}\right)}}$ & $1.27 \times 10^{-2}$ & $4.79 \times 10^{-3}$ & $2.02 \times 10^{-3}$ & $9.11 \times 10^{-4}$ & $4.29 \times 10^{-4}$ \\
$\left\|L y_{h}\right\|_{L^{2}\left(Q_{T}\right)}$ & 3.86 & 3.45 & 3.36 & 3.85 & 4.16 \\
$\left\|\lambda_{h}\right\|_{L^{2}\left(Q_{T}\right)}$ & $6.37 \times 10^{-6}$ & $1.65 \times 10^{-6}$ & $3.88 \times 10^{-7}$ & $9.74 \times 10^{-8}$ & $2.90 \times 10^{-8}$ \\
$\kappa$ & $2.02 \times 10^{8}$ & $2.62 \times 10^{9}$ & $2.05 \times 10^{10}$ & $1.61 \times 10^{11}$ & $1.32 \times 10^{12}$ \\
$\operatorname{card}\left(\left\{\lambda_{h}\right\}\right)$ & 554 & 2135 & 8381 & 33209 & 132209 \\
$\sharp$ CG iterates & 141 & 331 & 720 & 1446 & 3318 \\
\hline
\end{tabular}

Table 11: Observation domain $q_{T}^{2}$. Example EX2 - $r=1-T=2-\|y\|_{L^{2}\left(q_{T}\right)}=2.75 \times 10^{-1}-$ $\|y\|_{L^{2}\left(Q_{T}\right)}=5.87 \times 10^{-1}$.

The exact solution $y$ corresponding to initial data (EX2) is displayed in Figure 3 (a) using the third mesh of the domain in Figure 2 (b). Figure 3 (b) illustrates the solution $y_{h}$ of the mixed formulation (41), where the observation $y_{o b s}$ is obtained as the restriction of $y$ to $q_{T}^{2}$.

\subsection{Two-dimensional space case $(N=2)$}

We now illustrate the method introduced in Section 2 in the two-dimensional case. The procedure is similar but a bit more involved on a computational point of view, since $Q_{T}$ is now a subset of $\mathbb{R}^{3}$.

In order to approach the mixed-formulation (7), we consider a mesh $\mathcal{T}_{h}$ of the domain $Q_{T}=$ $\Omega \times(0, T)$ formed by triangular prisms. This mesh is obtained by extrapolating along the time axis a triangulation of the spatial domain $\Omega$. For an example in the case $\Omega=(0,1)^{2}$ and $T=2$ see Figure 4 (b) and for an example in the case of non-rectangular domains $\Omega \subset \mathbb{R}^{2}$ see Figure 5 (b). For both examples, the extrapolation along the the time axis is uniform : the height of the prismatic elements $\Delta t$ is constant. 


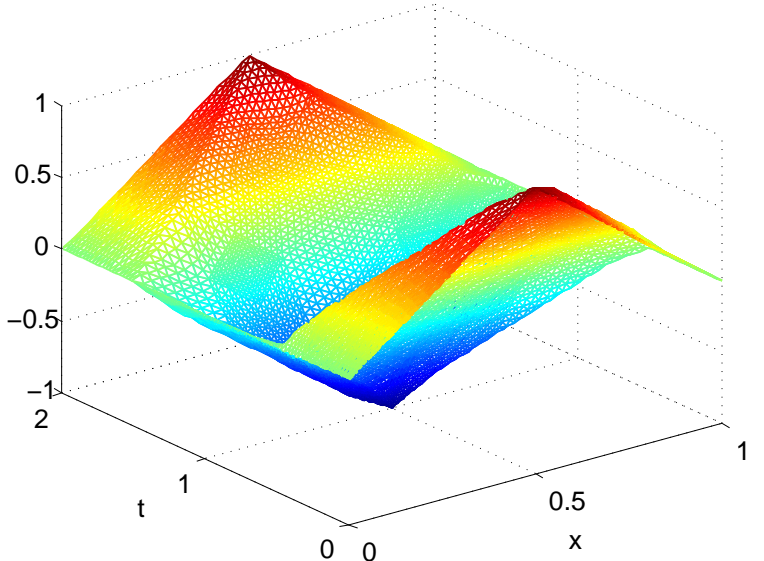

(a)

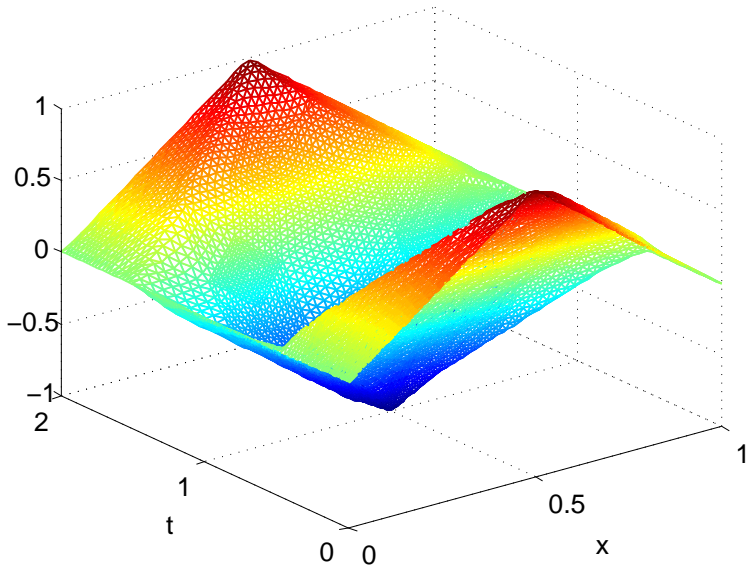

(b)

Figure 3: Example (EX2) (a) Reference solution. (b) Solution reconstructed from the observation $y_{o b s}=\left.y\right|_{q_{T}^{2}}$.

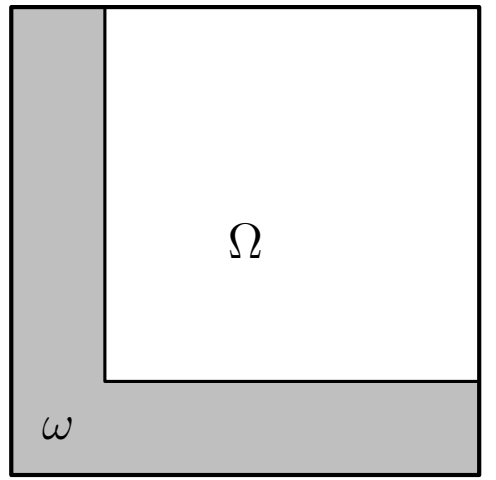

(a)

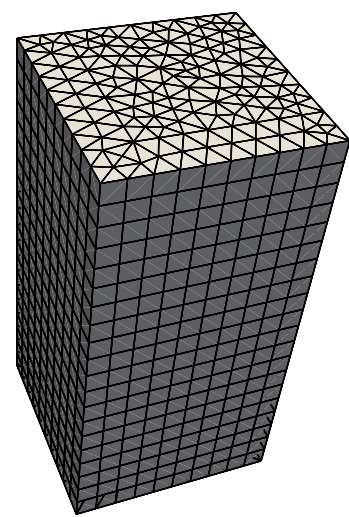

(b)

Figure 4: (a) Example of sets $\Omega$ and $\omega$. (b) Example of mesh for $\Omega=(0,1)^{2}$ and $T=2$.

Let $Z_{h}$ be the finite dimensional space defined as follows

$$
Z_{h}=\left\{\begin{array}{l|l}
\varphi_{h}=\psi\left(x_{1}, x_{2}\right) \theta(t) \in C^{1}\left(Q_{T}\right) & \begin{array}{l}
\left.\psi\right|_{K_{x_{1} x_{2}}} \in \mathbb{P}\left(K_{x y}\right),\left.\theta\right|_{K_{t}} \in \mathbb{Q}\left(K_{t}\right) \\
\text { for every } K=K_{x_{1} x_{2}} \times K_{t} \in \mathcal{T}_{h}
\end{array}
\end{array}\right\}
$$

$\mathbb{P}\left(K_{x_{1} x_{2}}\right)$ is the space of functions corresponding to the reduced Hsieh-Clough-Tocher (HCT for short) $C^{1}$-element recalled in Section 4.1.1; $\mathbb{Q}\left(K_{t}\right)$ is a space of degree three polynomials on the interval $K_{t}$ of the form $\left[t_{j}, t_{j+1}\right]$ defined uniquely by their value and the value of their first derivative at the point $t_{j}$ and $t_{j+1}$. In other words, $Y_{h}$ is the finite element space obtained as a tensorial product between the reduced HCT finite element and cubic Hermite finite element. We check that on each element $K=K_{x_{1} x_{2}} \times K_{t}$, the function $\varphi_{h}$ is determined uniquely in term of the values of $\Sigma_{K}:=\left\{\varphi\left(a_{i}\right), \varphi_{x_{1}}\left(a_{i}\right), \varphi_{x_{2}}\left(a_{i}\right), \varphi_{t}\left(a_{i}\right), \varphi_{x_{1}, t}\left(a_{i}\right), \varphi_{x_{2}, t}\left(a_{i}\right), i=1, \cdots, 6\right\}$ at the six nodes $a_{i}$ of $K$. Therefore, $\operatorname{dim} \Sigma_{K}=36$.

Similarly, let $\Lambda_{h}$ be the finite dimensional space defined by

$$
\Lambda_{h}=\left\{\begin{array}{l|l}
\varphi_{h}=\psi\left(x_{1}, x_{2}\right) \theta(t) \in C^{0}\left(Q_{T}\right) & \begin{array}{l}
\left.\psi\right|_{K_{x_{1} x_{2}}} \in \mathbb{P}_{1}\left(K_{x_{1} x_{2}}\right),\left.\theta\right|_{K_{t}} \in \mathbb{Q}_{1}\left(K_{t}\right) \\
\text { for every } K=K_{x_{1} x_{2}} \times K_{t} \in \mathcal{T}_{h}
\end{array}
\end{array}\right\}
$$


where $\mathbb{P}_{1}\left(K_{x_{1} x_{2}}\right)$ and $\mathbb{Q}_{1}\left(K_{t}\right)$ are the spaces of degree one polynomials on the triangle $K_{x_{1} x_{2}}$ and interval $K_{t}$ respectively.

For any $h$, we check that $Z_{h} \subset Z$ and that $\Lambda_{h} \subset \Lambda$.

\subsubsection{Wave equation in a square}

We first consider the case $\Omega$ defined by the unit square and again some explicit solutions used in 8. Precisely, we define the following smooth initial condition:

$$
\left(\mathbf { E X 1 - 2 D ) } \left\{\begin{array}{l}
y_{0}\left(x_{1}, x_{2}\right)=256 x_{1}^{2} x_{2}^{2}\left(1-x_{1}\right)^{2}\left(1-x_{2}\right)^{2} \\
y_{1}\left(x_{1}, x_{2}\right)=\left(1-\left|2 x_{1}-1\right|\right)\left(1-\left|2 x_{2}-1\right|\right)
\end{array} \quad\left(x_{1}, x_{2}\right) \in \Omega\right.\right.
$$

The corresponding solution of (1) with $c \equiv 1, d \equiv 0$ and $f \equiv 0$ is given by :

$$
y\left(x_{1}, x_{2}, t\right)=\sum_{k, l>0}\left(a_{k l} \cos \left(\mu_{k l} t\right)+\frac{b_{k l}}{\mu_{k l}} \sin \left(\mu_{k l} t\right)\right) \sin (k \pi x) \sin (l \pi y),
$$

where $\mu_{k l}=\pi \sqrt{k^{2}+l^{2}}$ for every $k, l \in \mathbb{Z}^{*}$ and

$$
\begin{aligned}
a_{k l} & =2^{10} \frac{\left(\pi^{2} k^{2}-12\right)\left(\pi^{2} l^{2}-12\right)}{\pi^{10} k^{5} l^{5}}\left((-1)^{k}-1\right)\left((-1)^{l}-1\right) \\
b_{k l} & =\frac{2^{5}}{\pi^{4} k^{2} l^{2}} \sin \frac{\pi k}{2} \sin \frac{\pi l}{2} .
\end{aligned}
$$

We also define the following initial data $\left(y_{0}, y_{1}\right) \in H_{0}^{1}(\Omega) \times L^{2}(\Omega)$ :

$$
\left(\mathbf { E X 2 - 2 D ) } \left\{\begin{array}{l}
y_{0}\left(x_{1}, x_{2}\right)=\left(1-\left|2 x_{1}-1\right|\right)\left(1-\left|2 x_{2}-1\right|\right) \\
y_{1}\left(x_{1}, x_{2}\right)=\mathbf{1}_{\left(\frac{1}{3}, \frac{2}{3}\right)^{2}}\left(x_{1}, x_{2}\right)
\end{array} \quad\left(x_{1}, x_{2}\right) \in \Omega .\right.\right.
$$

The Fourier coefficients of the corresponding solution are

$$
\begin{aligned}
a_{k l} & =\frac{2^{5}}{\pi^{4} k^{2} l^{2}} \sin \frac{\pi k}{2} \sin \frac{\pi l}{2} \\
b_{k l} & =\frac{1}{\pi^{2} k l}\left(\cos \frac{\pi k}{3}-\cos \frac{2 \pi k}{3}\right)\left(\cos \frac{\pi l}{3}-\cos \frac{2 \pi l}{3}\right) .
\end{aligned}
$$

In what follows, we consider $\omega$ the subset of $\Omega$ described in Figure 4 (a) and given by:

$$
\omega=((0,0.2) \times(0,1)) \cup((0,1) \times(0,0.2)) .
$$

It is easy to see that this choice of $\omega$ and $T=2$ provide a domain $q_{T}=\omega \times(0, T)$ which satisfies the geometric optic condition, and, hence, inequality $(\mathcal{H}$ ) holds. We consider 3 levels of meshes of $Q_{T}$, labelled from 1 to 3 and containing the number of elements (prisms) and nodes listed in Table 12.

\begin{tabular}{|c|ccc|}
\hline Mesh Number & 1 & 2 & 3 \\
\hline Number of elements & 5320 & 15320 & 42230 \\
Number of nodes & 3234 & 8799 & 23370 \\
$\Delta t$ & 0.2 & 0.1 & 0.05 \\
\hline
\end{tabular}

Table 12: Characteristics of the meshes used for $Q_{T}=(0,1)^{2} \times(0,2)$.

For each of these meshes we solve the mixed formulation (7) with the term $y_{\text {obs }}$ appearing in the right-hand side obtained as the restriction to $q_{T}$ of the solution computed by (76) for initial data EX1-2D and EX2-2D. 


\begin{tabular}{|c|ccc|}
\hline Mesh number & 1 & 2 & 3 \\
\hline$\frac{\left\|y-y_{h}\right\|_{L^{2}\left(Q_{T}\right)}}{\|y\|_{L^{2}\left(Q_{T}\right)}}$ & $4.58 \times 10^{-2}$ & $3.18 \times 10^{-2}$ & $1.38 \times 10^{-2}$ \\
$\left\|L y_{h}\right\|_{L^{2}\left(Q_{T}\right)}$ & 1.44 & 1.05 & 1.05 \\
$\left\|\lambda_{h}\right\|_{L^{2}\left(Q_{T}\right)}$ & $2.87 \times 10^{-5}$ & $1.36 \times 10^{-5}$ & $7.34 \times 10^{-6}$ \\
$\sharp \mathrm{CG}$ iterates & 121 & 180 & 168 \\
\hline
\end{tabular}

Table 13: $\varepsilon=0$ : Example EX1-2D $-r=1$.

Table 13 concerns the example EX1-2D. In this table we list the norm of the relative error between the exact solution $y$ given by $(76)$ and the solution $y_{h}$ of the mixed formulation (7), the $L^{2}$ norm of $L y_{h}$ and the $L^{2}$ norm of the Lagrange multiplier $\lambda_{h}$.

As theoretically stated in Remark 1 and observed in numerical experiments in the case $N=1$ (see, for instance, Table 13, the Lagrange multiplier $\lambda_{h}$ vanishes as $h \rightarrow 0$. In Table 14 we display the results obtained by numerically solving the variational problem (7) obtained from the mixed formulation when $\lambda_{h}=0$.

\begin{tabular}{|c|ccc|}
\hline Mesh number & 1 & 2 & 3 \\
\hline$\frac{\left\|y-y_{h}\right\|_{L^{2}\left(Q_{T}\right)}}{\|y\|_{L^{2}\left(Q_{T}\right)}}$ & $7.05 \times 10^{-2}$ & $4.44 \times 10^{-2}$ & $2.37 \times 10^{-2}$ \\
$\left\|L y_{h}\right\|_{L^{2}\left(Q_{T}\right)}$ & 1.31 & 0.97 & 0.97 \\
\hline
\end{tabular}

Table 14: Example EX1-2D $-r=1-\lambda$ fixed to zero.

Tables 15 and 16 display the results obtained for the initial data specified by $\mathbf{E X 2 - 2 D}$, for the solutions $\left(y_{h}, \lambda_{h}\right)$ of the mixed formulation and for the variational problem obtained when $\lambda_{h}=0$ respectively.

\begin{tabular}{|c|ccc|}
\hline Mesh number & 1 & 2 & 3 \\
\hline$\frac{\left\|y-y_{h}\right\|_{L^{2}\left(Q_{T}\right)}}{\|y\|_{L^{2}\left(Q_{T}\right)}}$ & $4.74 \times 10^{-2}$ & $3.72 \times 10^{-2}$ & $2.09 \times 10^{-2}$ \\
$\left\|L y_{h}\right\|_{L^{2}\left(Q_{T}\right)}$ & 1.18 & 0.89 & 1.06 \\
$\left\|\lambda_{h}\right\|_{L^{2}\left(Q_{T}\right)}$ & $3.21 \times 10^{-5}$ & $1.46 \times 10^{-5}$ & $1.17 \times 10^{-5}$ \\
$\sharp \mathrm{CG}$ iterates & 128 & 191 & 168 \\
\hline
\end{tabular}

Table 15: Example EX2-2D $-r=1$.

The results are similar for both examples. In both cases we observe a linear convergence of $y_{h}$ to $y$ in the norm $L^{2}$ over $Q_{T}$ when $h$ goes to zero. Similarly, the norm $\left\|\lambda_{h}\right\|_{L^{2}\left(Q_{T}\right)}$ linearly decreases as $h$ goes to zero.

\begin{tabular}{|c|ccc|}
\hline Mesh number & 1 & 2 & 3 \\
\hline$\frac{\left\|y-y_{h}\right\|_{L^{2}\left(Q_{T}\right)}}{\|y\|_{L^{2}\left(Q_{T}\right)}}$ & $6.75 \times 10^{-2}$ & $4.93 \times 10^{-2}$ & $3.37 \times 10^{-2}$ \\
$\left\|L y_{h}\right\|_{L^{2}\left(Q_{T}\right)}$ & 1.07 & 0.82 & 0.97 \\
\hline
\end{tabular}

Table 16: Example EX2-2D $-r=1-\lambda$ fixed to zero.

\subsubsection{Wave equation in a non-rectangular domain of $\mathbb{R}^{2}$}

Let $\Omega \subset \mathbb{R}^{2}$ be a domain with a regular boundary and $\omega$ a non-empty subset with regular boundary. An example of such a configuration is illustrated in Figure 5 (a). As in the previous section, we take $T=2$ and we build a mesh formed by triangular prisms of the domain $Q_{T}=\Omega \times(0, T)$. An 
example of such a mesh associated to the domain $\Omega$ is displayed in Figure 5 (b). This mesh is composed by 17934 nodes distributed in 32140 prismatic elements (this mesh corresponds to the mesh number 2 described in Table 17 .

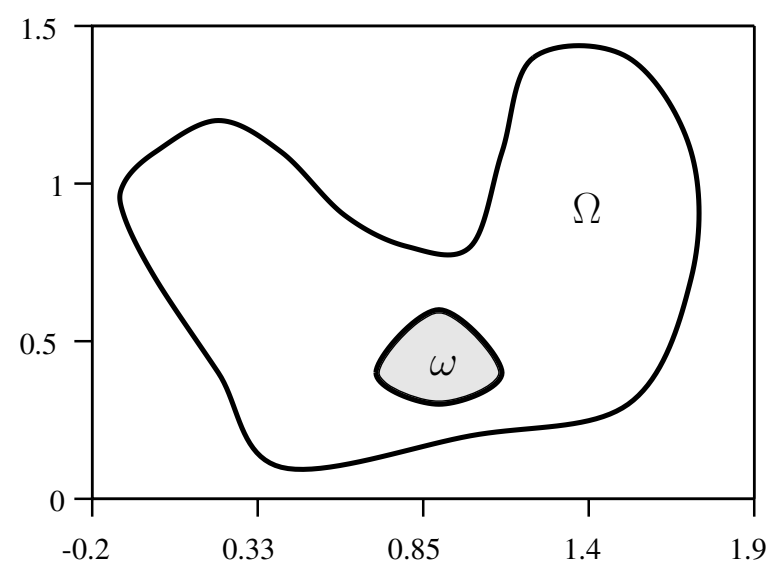

(a)

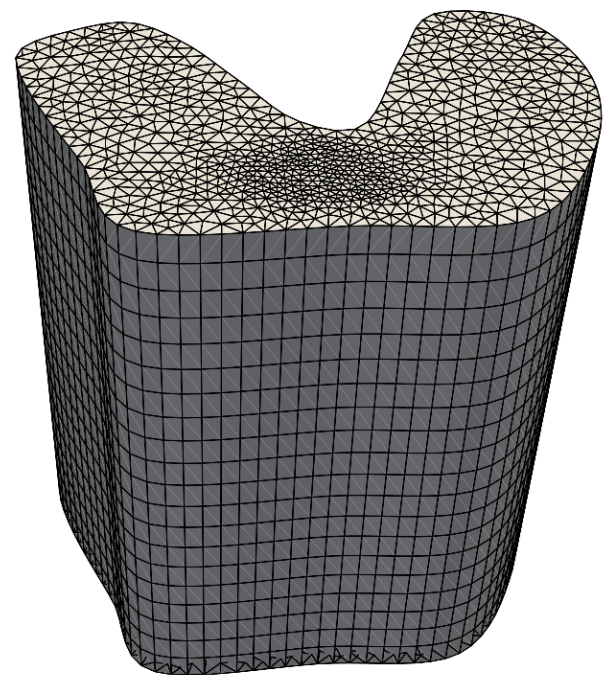

(b)

Figure 5: (a) Example of sets $\Omega$ and $\omega$. (b) Example of mesh of the domain $Q_{T}$.

We consider three levels of meshes of the domain $Q_{T}$ formed by the number of prisms and containing the number of nodes reported in Table 17.

\begin{tabular}{|c|ccc|}
\hline Mesh number & 1 & 2 & 3 \\
\hline Number of elements & 5730 & 321400 & 130280 \\
Number of nodes & 3432 & 17934 & 69864 \\
Height of elements $(\Delta t)$ & 0.2 & 0.1 & 0.05 \\
\hline
\end{tabular}

Table 17: Characteristics of the three meshes associated with $Q_{T}$.

Comparing to the situation described in Subsection 5.2.1, the eigenfunctions and eigenvectors of the Dirichlet Laplace operator defined on $\Omega$ are not explicitly available here. Consequently, from a given set of initial data, we numerically solve the wave equation (1) using a standard timemarching method, from which we can extract an observation on $q_{T}$. Precisely, we use a $P_{1}$ finite elements method in space coupled with a Newmark unconditionally stable scheme for the time discretization. Hence, we solve the wave equation on the same mesh which was extrapolated in time in order to obtain the mesh number 2 of $Q_{T}$. This two-dimensional mesh contains 1704 nodes and 3257 triangles. The time discretization step is $\Delta t=10^{-2}$. We denote $\bar{y}_{h}$ the solution obtained in this way for the initial data $\left(y_{0}, y_{1}\right) \in H_{0}^{1}(\Omega) \times L^{2}(\Omega)$ given by

$$
\left\{\begin{array}{ll}
-\Delta y_{0}=10, & \text { in } \Omega \\
y_{0}=0, & \text { on } \partial \Omega,
\end{array} y_{1}=0 .\right.
$$

From $\bar{y}_{h}$ we generate the observation $y_{o b s}$ as the restriction of $\bar{y}_{h}$ to $q_{T}$. Finally, from this observation we reconstruct $y_{h}$ as the solution of the mixed formulation 290 on each of the three 
meshes described in Table 17. Table 18 display some norms of $y_{h}$ and $\lambda_{h}$ obtained for the three meshes and illustrates again the convergence of the method.

\begin{tabular}{|c|ccc|}
\hline Mesh number & 1 & 2 & 3 \\
\hline$\frac{\left\|\bar{y}_{h}-y_{h}\right\|_{L^{2}\left(Q_{T}\right)}}{\left\|\bar{y}_{h}\right\|_{L^{2}\left(Q_{T}\right)}}$ & $1.88 \times 10^{-1}$ & $8.04 \times 10^{-2}$ & $7.11 \times 10^{-2}$ \\
$\left\|L y_{h}\right\|_{L^{2}\left(Q_{T}\right)}$ & 3.21 & 2.01 & 1.57 \\
$\left\|\lambda_{h}\right\|_{L^{2}\left(Q_{T}\right)}$ & $8.26 \times 10^{-5}$ & $3.62 \times 10^{-5}$ & $2.84 \times 10^{-5}$ \\
$\sharp$ CG iterates & 52 & 167 & 400 \\
\hline
\end{tabular}

Table 18: Initial data $\left(y_{0}, y_{1}\right)$ given by $(79)-r=1$.

Figure 6(a) displays the solution $y_{0}$ of 79 and Figure 6(b) displays the initial position $y_{h}(\cdot, 0)$ corresponding to the solution of our inverse problem. The error between these two functions is given by $\left\|y_{0}-y_{h}(\cdot, 0)\right\|_{L^{2}(\Omega)}=2.05 \times 10^{-2}$ which is consistent with the results reported in Table 18 .

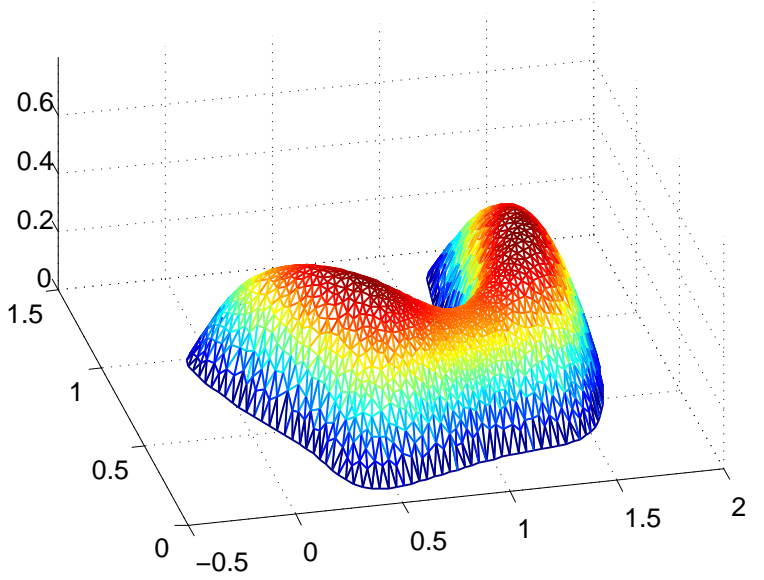

(a)

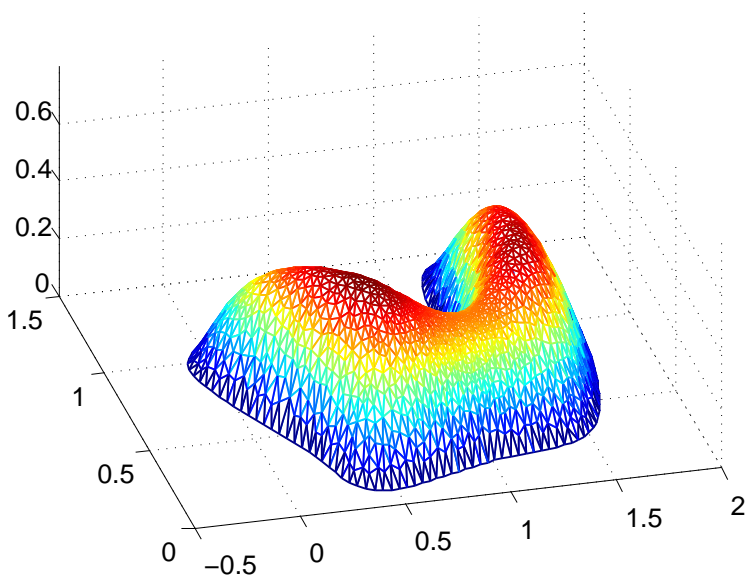

(b)

Figure 6: (a) Initial data $y_{0}$ given by (79). (b) Reconstructed initial data $y_{h}(\cdot, 0)$.

\section{Concluding remarks and perspectives}

The mixed formulations we have introduced here in order to address inverse problems for the wave equation seems original. These formulations are nothing else than the Euler systems associated to least-squares type functionals and depend on both the state to be reconstruct and a Lagrange multiplier. This Lagrange multiplier is introduced to take into account the state constraint $L y-f=$ 0 and turns out to be the controlled solution of a wave equation with the source term $\left(y-y_{o b s}\right) 1_{q_{T}}$. This approach, recently used in a controllability context in [12, leads to a variational problem defined over time-space functional Hilbert spaces, without distinction between the time and the space variable. The main ingredient allowing to prove the well-posedness of the mixed formulation and therefore the reconstruction of the solution, is a generalized observability inequality, assuming here some geometric conditions on the observation zone.

At the practical level, the discrete mixed time-space formulation is solved in a systematic way in the framework of the finite element theory. The approximation is conformal allowing to obtain the strong convergence of the approximation as the discretization parameters tends to zero. In particular, we emphasize that there is no need, contrarily to the classical approach, to prove some 
uniform discrete observability inequality: we simply use the observability equality on the finite dimensional discrete space. The resolution amounts to solve a sparse symmetric linear system : the corresponding matrix can be preconditioned if necessary, and may be computed once for all as it does not depend on the observation $y_{o b s}$. Eventually, the space-time discretization of the domain allows an adaptation of the mesh so as to reduce the computational cost and capture the main features of the solutions. Similarly, this space-time formulation is very appropriate to the non-cylindrical situation.

In agreement with the theoretical convergence, the numerical experiments reported here display a very good behavior and robustness of the approach: the reconstructed approximate solution converges strongly to the solution of the wave equation associated to the available observation. Remark that from the continuous dependence of the solution with respect to the observation, the method is robust with respect to the possible noise on the data.

As mentioned at the end of Section 3, additional assumption on the source term allows to determine uniquely the pair $(y, f)$ from a partial measurement on $q_{T}$ or on a part $\Sigma_{T}$ sufficiently large of the boundary. For instance, from [25, Theorem 2.1], assuming that the source term takes the form $f(x, t)=\sigma(t) \mu(x)$ with $\sigma \in C^{1}([0, T]), \sigma(0) \neq 0$ and $\mu \in H^{-1}(\Omega)$, then the following holds: there exists a positive constant $C$ such that

$$
\|\mu\|_{H^{-1}(\Omega)}^{2} \leq C\left(\left\|\frac{\partial y}{\partial \nu}\right\|_{L^{2}\left(\Sigma_{T}\right)}^{2}+\|L y-\sigma(t) \mu(x)\|_{L^{2}\left(Q_{T}\right)}^{2}\right), \quad \forall(y, \mu) \in S
$$

where $y$ solves (1) with $\left(y_{0}, y_{1}\right) \equiv 0, c=1$ and $\left(\Sigma_{T}, T, Q_{T}\right)$ satisfies a geometric condition and $S$ denotes an appropriate functional space. Using this inequality (similar to $\mathcal{H}$ ), we can study the mixed formulation associated to the Lagrangian from $S \times L^{2}\left(Q_{T}\right) \rightarrow \mathbb{R}$ defined by

$$
\mathcal{L}((y, \mu), \lambda):=\frac{1}{2}\left\|\frac{\partial y}{\partial \nu}-y_{o b s}\right\|_{L^{2}\left(\Sigma_{T}\right)}^{2}+\int_{Q_{T}} \lambda(L y-\sigma \mu) d x d t
$$

to fully reconstruct $y$ and $\mu$ from $y_{o b s}$ and $\sigma$.

Eventually, since the mixed formulations rely essentially on a generalized observability inequality, it may be employed to any other observable systems for which such property is available : we mention notably the parabolic case usually - in view of regularization property - badly conditioned and for which direct and robust methods are certainly very advantageous. We refer to [23] where this issue is investigated.

\section{References}

[1] C. Bardos, G. Lebeau, and J. Rauch, Sharp sufficient conditions for the observation, control, and stabilization of waves from the boundary, SIAM J. Control Optim., 30 (1992), pp. 1024-1065.

[2] L. Beilina and M. V. Klibanov, Approximate Global Convergence and Adaptivity for coefficient inverse problems, Springer US, 2012.

[3] M. Bernadou and K. Hassan, Basis functions for general Hsieh-Clough-Tocher triangles, complete or reduced, Internat. J. Numer. Methods Engrg., 17 (1981), pp. 784-789.

[4] D. Boffi, F. Brezzi, And M. Fortin, Mixed finite element methods and applications, vol. 44 of Springer Series in Computational Mathematics, Springer, Heidelberg, 2013.

[5] L. Bourgeois And J. Dardé, A duality-based method of quasi-reversibility to solve the Cauchy problem in the presence of noisy data, Inverse Problems, 26 (2010), pp. 095016, 21. 
[6] L. Bourgeois And J. DARdÉ, A quasi-reversibility approach to solve the inverse obstacle problem, Inverse Probl. Imaging, 4 (2010), pp. 351-377.

[7] C. Castro, N. Cîndea, And A. MÜnch, Controllability of the linear one-dimensional wave equation with inner moving forces, SIAM J. Control Optim., 52 (2014), pp. 4027-4056.

[8] D. Chapelle, N. Cîndea, and P. Moireau, Improving convergence in numerical analysis using observers - the wave-like equation case, Math. Models Methods Appl. Sci., 22 (2012), pp. 1250040, 35 .

[9] P. G. Ciarlet, The finite element method for elliptic problems, vol. 40 of Classics in Applied Mathematics, Society for Industrial and Applied Mathematics (SIAM), Philadelphia, PA, 2002. Reprint of the 1978 original [North-Holland, Amsterdam; MR0520174 (58 \#25001)].

[10] N. Cîndea, E. Fernández-Cara, And A. Münch, Numerical controllability of the wave equation through primal methods and carleman estimates, ESAIM Control Optim. Calc. Var., 19 (2013), pp. 1076-1108.

[11] N. Cîndea, A. Imperiale, and P. Moireau, Data assimilation of time under-sampled measurements using observers, the wave-like equation example, ESAIM Control Optim. Calc. Var., (2014).

[12] N. Cîndea And A. Münch, A mixed formulation for the direct approximation of the control of minimal $L^{2}$-norm for linear type wave equations, To appear in Calcolo (http://hal.archivesouvertes.fr/hal-00853767).

[13] C. Clason and M. V. Klibanov, The quasi-reversibility method for thermoacoustic tomography in a heterogeneous medium, SIAM J. Sci. Comput., 30 (2007/08), pp. 1-23.

[14] J. W. Daniel, The approximate minimization of functionals, Prentice-Hall Inc., Englewood Cliffs, N.J., 1971.

[15] D. A. Dunavant, High degree efficient symmetrical Gaussian quadrature rules for the triangle, Internat. J. Numer. Methods Engrg., 21 (1985), pp. 1129-1148.

[16] M. Fortin And R. Glowinski, Augmented Lagrangian methods, vol. 15 of Studies in Mathematics and its Applications, North-Holland Publishing Co., Amsterdam, 1983. Applications to the numerical solution of boundary value problems, Translated from the French by B. Hunt and D. C. Spicer.

[17] R. GLowinski, J.-L. Lions, And J. He, Exact and approximate controllability for distributed parameter systems, vol. 117 of Encyclopedia of Mathematics and its Applications, Cambridge University Press, Cambridge, 2008. A numerical approach.

[18] M. V. Klibanov and A. Timonov, Carleman estimates for coefficient inverse problems and numerical applications, Inverse and Ill-posed Problems Series, VSP, Utrecht, 2004.

[19] V. Komornik and P. Loreti, Observability of discretized wave equations, Bol. Soc. Parana. Mat. (3), 25 (2007), pp. 67-76.

[20] J.-L. Lions, Contrôlabilité exacte, perturbations et stabilisation de systèmes distribués. Tome 1, vol. 8 of Recherches en Mathématiques Appliquées [Research in Applied Mathematics], Masson, Paris, 1988. Contrôlabilité exacte. [Exact controllability], With appendices by E. Zuazua, C. Bardos, G. Lebeau and J. Rauch. 
[21] A. MEYER, A simplified calculation of reduced hct-basis functions in a finite element context, Comput. Methods Appl. Math., 12 (2012), pp. 486-499.

[22] A. MüNCH, A uniformly controllable and implicit scheme for the 1-D wave equation, M2AN Math. Model. Numer. Anal., 39 (2005), pp. 377-418.

[23] A. MüNCH AND D. SouzA, Inverse problems for linear parabolic equation via weighted mixed formulations, In preparation.

[24] K. Ramdani, M. Tucsnak, and G. Weiss, Recovering and initial state of an infinitedimensional system using observers, Automatica J. IFAC, 46 (2010), pp. 1616-1625.

[25] M. Yамамото AND X. Zhang, Global uniqueness and stability for an inverse wave source problem for less regular data, J. Math. Anal. Appl., 263 (2001), pp. 479-500. 